\title{
Learning about Monetary Policy Rules when the Cost Channel Matters
}

\author{
Gonzalo Llosa* y Vicente Tuesta** \\ * UCLA \\ ** Banco Central de Reserva del Perú
}

\author{
DT. N ${ }^{\circ} 2007-014$ \\ Serie de Documentos de Trabajo \\ Working Paper series \\ Agosto 2007
}

\begin{abstract}
Los puntos de vista expresados en este documento de trabajo corresponden a los de los autores y no reflejan necesariamente la posición del Banco Central de Reserva del Perú.
\end{abstract}

The views expressed in this paper are those of the authors and do not reflect necessarily the position of the Central Reserve Bank of Peru. 


\title{
"Learning about Monetary Policy Rules when the Cost Channel Matters"*
}

\author{
Luis-Gonzalo Llosa ${ }^{\dagger}$ \\ UCLA
}

\author{
Vicente Tuesta ${ }^{\ddagger}$ \\ Banco Central de Reserva del Perú
}

First version, February 2007, this version, September 2007

\begin{abstract}
We study how determinacy and expectational stability (E-stability) of rational expectations equilibrium may be affected by monetary policy when the cost channel of monetary policy matters. We focus on both instrumental Taylor-type rules and optimal target rules. We show that standard instrument rules can easily induce indeterminacy and expectational instability when the cost channel is present. Overall, a naïve application of the traditional Taylor principle in this setting could be misleading. Regarding optimal rules, we find that "expectational-based rules" do not always induce determinate and E-stable equilibrium. This result stands in contrast to the findings of Evans and Honkapohja (2003) for the baseline "New Keynesian" model. Yet, a policy that it is a source of instability under learning in the baseline new keynesian model, i.e. "fundamental rule" under commitment, is a possible antidote when the cost channel is active.
\end{abstract}

Keywords: Learning; Monetary Policy Rules; Cost Channel; Indeterminacy.

JEL classification: E4; E5; F31: F41

\footnotetext{
${ }^{*}$ We are grateful to Guillermo Calvo and seminar participants at the 13th Conference of Computation and Finance held in HEC Montreal for helpful suggestions and comments. The views expressed in this paper are those of the authors and do not necessarily reflect the views of the Banco Central de Reserva del Perú. Any errors are our own responsibility.

${ }^{\dagger}$ Economics Department, UCLA E-mail address: luisllosa@ucla.edu

${ }^{\ddagger}$ Correspondence author. Research Department, Banco Central de Reserva del Perú, Jr. Miroquesada \#441, Lima 01, Peru. tel: (51 1) 613 2000, E-mail address: vicente.tuesta@bcrp.gob.pe.
} 


\section{Introduction}

There is growing and recent empirical evidence showing hat the cost channel of monetary policy, i.e. when the interest rate affects directly firm's price setting behavior, has important implications in both inflation dynamics and the design of optimal monetary policy. Ravenna and Walsh (2006, RW) and Chowdhury et. al. (2006) provide empirical evidence for the cost channel in the U.S. and the Euro Area, respectively. Barth and Ramey (2001) find a significant cost channel effect on U.S. data at industry level. Christiano, Eichenbaum and Evans (2005) estimate a DSGE model of the U.S. economy and find that monetary policy operates also through the supply side. From the normative point of view, RW show the effects of the cost channel in terms of monetary policy and find that a trade-off between stabilizing inflation and output arises endogenously as a consequence of the cost channel.

At the same time, a recent ongoing literature has begun to evaluate the stability under learning of rational expectations equilibrium (REE) in New Keynesian Models. Using the standard New Keynesian Framework (or baseline model) in which the cost channel is absent, Bullard and Mitra (2002, BM hereafter) find that the determinacy and learnability of a variety of instrument rules is guaranteed if the traditional Taylor principle is satisfied, i.e. the interest rate reacts more than one-for-one to inflation (also referred as active rules). ${ }^{1}$ In the same framework, Evans and Honkapohja (2003, 2006, hereafter EH) show that optimal target rules (under discretion or commitment) renders the REE always unstable under learning if the optimal policy rule is derived based on the assumption that agents have rational expectations (referred as "fundamental-based rule"). EH proposed an alternative implementation of the optimal rule by relaxing the assumption of rational expectations on private agents, referred as "expectation-based optimal rule", and find that this type of rules (which react optimally to private sector expectations) can always induce determinacy and learnability. EH's economic intuition relies on the fact that their proposed "expectation-based optimal rule" always satisfies the Taylor principle. Yet, the implications of the cost channel in terms of determinacy and stability under adaptive learning (also referred as learnability) of the rational expectations equilibrium (REE) have been left aside in the discussion. ${ }^{2}$

This paper examines the effects of the cost channel coupled with a variety of instrument and optimal target rules on the determinacy (i.e. a unique and non-explosive) and learnability conditions of the REE. Particularly, we study local determinacy and E-stability properties of

\footnotetext{
${ }^{1}$ Throughout the paper we will refer to those rules satisfying the traditional Taylor principle as active rules. Otherwise, we will refer to passive rules.

${ }^{2}$ As Bullard (2006) pointed out, since adaptive learning is a "minimal deviation from rational expectations", its stability should be viewed as an additional minimal criterion, besides determinacy, that a REE equilibrium should meet.
} 
the REE in the cost channel model proposed by RW (2006). ${ }^{34}$ In this sense, our work extends BM's (2002) and EH's (2003, 2006) baseline-economy results to a cost-channel framework. We perform the analysis of instrumental rules under two specifications. In the first one, the interest rate reacts to current values; this is called contemporaneous data specification. In the second one, the interest rate reacts to forward expectations; this is called forward-looking rule specification (also referred as forward expectations or forecast-based rules). In the case of target rules and in the same fashion of EH $(2003,2006)$, we analyze "fundamental-based" and "expectations-based" rules under both discretion and commitment.

Overall, our analytical findings show that the cost channel influences both determinacy and learnability conditions, making them more stringent with respect to the baseline new Keynesian model. In the case of the contemparaneous data instrument rule, our analytical condition for determinacy is consistent with that of E-stability. This condition can be interpreted as the long-run version of the Taylor principle which, as stated by Bullard and Mitra (2002) and Woodford (2003, Chapter 4, section 2), implies that in the long-run the nominal interest rate should be raised more than the increase in inflation. Yet, in the model with the cost channel its implications differ importantly from those of the baseline new Keynesian framework. In fact, in the baseline model the traditional Taylor principle implies its long-run version (that is if the Taylor rule has an inflation coefficient larger than one, it generates both determinacy and learnability) and therefore it is a sufficient condition for both determinacy and learnability. Instead, to the extent that the cost channel is present, the traditional Taylor principle might not imply its long-run version and therefore it becomes a necessary but not sufficient condition. This result is striking since standard policies recommended to the baseline new Keynesian framework (as in BM) to guarantee determinacy and E-stability may not be effective or could even be counterproductive if the cost channel is present.

Under forecast-based rules, a crucial result is that, unlike to BM (2002), the Taylor principle does not guarantee either a determinate or an E-stable equilibrium, i.e. besides the long-run taylor principle additional conditions are also required for both determinacy and learnability. Moreover, even with a null response to the output gap, if the cost channel is strong enough and the nominal interest rate is adjusted according to the traditional Taylor Principle (positively and more than one-for-one reaction to expected inflation) a determinate and E-stable REE is not necessarily attainable. This result rises doubts about the validity of the Taylor principle

\footnotetext{
${ }^{3}$ Evans and Honkapohja (1999, 2001) developed the criterion of Expectational Stability (or E-stability): the conditions under which agents are able to learn (through least squares) the reduced form dynamics under the assumption of rational expectations. E-stability therefore provides a robustness criterion: if agents make small mistakes in expectations relative to those consistent with the associated REE, then a policy rule that is E-stable ensures such mistakes are corrected over time.

${ }^{4}$ Even so learnability is a more general concept than E-stability, throughout the paper we will use both terms interchangeably.
} 
as a useful guideline for forward-looking instrument rules when the cost channel is present.

Under optimal policy rules, our results can be summarized as follows. First, under discretion, we find that a "fundamental-based" optimal policy rule, that is, an interest rate rule that reacts only to fundamental shocks, implies that the equilibrium is indeterminate and unstable in the learning dynamics, results that coincide with those of EH (2003). Following EH (2003) we also derive an "expectations-base" rule that might, allegedly, perform well in both grounds. In fact, EH (2003) show that if the central bank assumes that the private sector does not have (initially) rational expectations, the resulting optimal "expectations-based" interest rule, renders the equilibrium always determinate and E-stable. However, different from EH, we show that the implied "expectations-based" rule does not always lead to stability under learning dynamics. Second, under optimal commitment, we find that the "fundamental-based" policy can be E-stable for a given parameter arrangement, contrasting with EH (2006) who show that this class of optimal rules is never stable under learning. Hence, to the extent that the cost channel is present, the policymaker's ability to commit to an optimal policy when the policy reaction function depends on fundamentals and lagged endogenous values, could be sufficient to stabilize the economy ${ }^{5}$.

In summary, the presence of cost channel imposes some difficulties to the central bank in achieving a determinate and learnable equilibrium even if the traditional Taylor principle holds or the monetary authority optimally reacts to private sector expectations. Therefore, BM and EH's proposed resolutions might not always perform well.

Our paper contributes to an important strand literature that has been studying determinacy and E-stability when the supply-side effects of monetary policy matter. Brückner and Schabert (2003) only study determinacy and point out that the cost channel introduces an additional upper bound to the inflation reaction in the Taylor rule. Surico (2006) finds that if a central bank assigns positive weight to output fluctuations a model with cost channel is more prone to multiple equilibria (indeterminacy) relative to the standard one. ${ }^{6}$ Benhabib et. al. (2001) show that, depending on the way money is introduced (e.g. money in the production function), some forms of active monetary policy bring about indeterminacy. Kurozomi (2006) proves that even a small degree of non-separability between consumption and money balances in the utility function causes the Taylor rule to be much more likely to induce indeterminacy or E-instability. Particularly, Kurozomi's analysis show the traditional Taylor principle may render the REE to be indeterminate and E-unstable. ${ }^{7}$

\footnotetext{
${ }^{5}$ This finding conccurs with those of Duffy and Xiao (2005). They find that if one includes the interest rate deviations in the objective, E-stability can be achieved without requiring the central bank to react to private sector expectations.

${ }^{6}$ Surico (2006) performs only determinacy analysis of Taylor rules with smoothness in the interest rate. The author focuses on two specifications of Taylor rules, namely contemporaneous and lagged data.

${ }^{7}$ Kurozomi (2006) stresses on the fact that the traditional Taylor principle does not always implies its long
} 
The rest of the paper is organized as follows. Section 2 outlines RW's model and discusses its main differences with respect to the baseline model. Section 3 describes the analysis of determinacy and learning under instrumental and target rules. Section 4 concludes.

\section{The Simple Environment}

In this section we summarize the log-linearized version of the model presented in RW . The model can be summarized by the following equations (equations 27 and 28 in RW's paper):

$$
\begin{aligned}
& \pi_{t}=\kappa_{x} x_{t}+\beta E_{t} \pi_{t+1}+\delta \kappa i_{t}+\mu_{t} \\
& x_{t}=E_{t} x_{t+1}-\frac{1}{\sigma}\left(i_{t}-E_{t} \pi_{t+1}\right)
\end{aligned}
$$

$\kappa \equiv[(1-\theta \beta)(1-\theta) / \theta]$ and $\kappa_{x} \equiv \kappa(\eta+\sigma)$. Variable $x_{t}$ is the output gap, $\pi_{t}$ inflation and $i_{t}$ is the percentage point deviation of the nominal interest rate around its steady state value. In the model, $\mu_{t}$ represents the traditional cost-push shock and $E_{t}$ symbolizes the standard expectation operator. We implicitly base our analysis of learning and monetary policy on "Euler Equation" approach as it is suggested in Honkapohja, Mitra and Evans (2003). Therefore, throughout the paper we assume that our systems are valid under both rational expectations and learning. In this sense, the expectation operation is taken to describe aggregate behavior regardless of the precise nature of agents' expectation formation. ${ }^{8}$

Equation (1) is a short run aggregate supply $(A S)$ curve that relates inflation with the output gap and the nominal interest rate. The parameter $\beta$ denotes the discount factor and $\kappa_{x}$ captures the sensitivity of inflation to movements in the output gap that depends on deep parameters such as the degree of price stickiness captured by $\theta$ and the inverse of the elasticity of the labor supply $\eta$. Equation (2) is a $I S$ curve that relates the output gap inversely to the domestic interest rate and positively with the expected future output gap. In this equation $\frac{1}{\sigma}$ represents the intertemporal elasticity of substitution.

Note that the previous two-equation system differs from the standard new Keynesian model (see Woodford 2003) due to the presence of the nominal interest $i_{t}$ in the staggered price equation, i.e. the cost channel of monetary policy. The existence of the cost channel is justified if firms must borrow working capital from intermediaries (for further details see RW). Just for comparison we define $\delta$ which is a discrete variable that takes the value of 1 when there is cost channel and 0 where there is not (baseline model). We assume that $\mu_{t}$ evolves according to an

run version unless the central bank does not target the output gap.

${ }^{8}$ Recently, Preston (2005) has proposed an interesting reformulation of intertemporal behavior under learning in which agents are assumed to incorporate a "subjective version" of their intertemporal budget constraint into their behavior under learning. In this paper, we abstract from this approach. 
exogenous first order autoregressive process

$$
\mu_{t}=\rho \mu_{t-1}+\varepsilon_{t}
$$

where $\varepsilon_{t}$ is an i.i.d noise with variances $\sigma_{\varepsilon}^{2}$. Finally, $0 \leq \rho<1$ is the correlation parameter.

\section{Determinacy and E-stability}

We supplement equations (1) through (2) with a policy rule for the interest rate $i_{t}$ that represents the behavior of the monetary authority. Here we study interest rate policy rules which have been extensively studied in the literature: instrumental and target rules. The main question is whether such rules lead to determinacy and stability under adaptive learning. The follwing propositions will show that either the traditional Taylor principle or EH's proposal to the resolution of the instability under learning cannot be taken for granted when the cost channel matters.

\subsection{Instrumental Rules}

In this subsection, we consider Taylor type of rules being evaluated with both contemporaneous data and forward expectations as in BM (2002).

\subsubsection{Contemporaneous data in the Taylor Rule}

We first assume a simple Taylor type rule (see Taylor 1993) in which the central bank reacts to price inflation and the output gap

$$
i_{t}=\phi_{\pi} \pi_{t}+\phi_{x} x_{t}
$$

where $\phi_{\pi}$ and $\phi_{x}$ are non-negative which measure the degree of responsiveness of the policy interest rate to inflation and output gap, respectively.

Substituting the policy rule (4) into (1) and (2), we can write the model involving the two endogenous variables $x_{t}$ and $\pi_{t}$

$$
\begin{aligned}
y_{t} & =\Gamma+\Omega E_{t} y_{t+1}+k \mu_{t} \\
w_{t} & =\rho w_{t-1}+\varepsilon_{t}
\end{aligned}
$$


where $y_{t}=\left[\pi_{t}, x_{t}\right]^{\prime}, w_{t}=\mu_{t}, \Gamma=0$, and

$$
\Omega=\psi\left[\begin{array}{cc}
\sigma \beta+\kappa_{x}+\beta \phi_{x}+\delta \kappa \phi_{x} & \sigma\left(\kappa_{x}+\delta \kappa \phi_{x}\right) \\
1-\beta \phi_{\pi}-\kappa \phi_{\pi} & \sigma\left(1-\delta \kappa \phi_{\pi}\right)
\end{array}\right]
$$

with $\psi=\left(\sigma+\phi_{x}+\kappa_{x} \phi_{\pi}-\delta \kappa \sigma \phi_{\pi}\right)^{-1}$.

Determinacy is analyzed by asking under which conditions $\Omega$ has both of its eigenvalues inside the unit circle. ${ }^{9}$ Propositions 1 summarizes the necessary and sufficient conditions for determinacy.

Proposition 1. Under contemporaneous data interest rate rules the necessary and sufficient conditions for a rational expectations equilibrium to be determinate are that

$$
\begin{aligned}
\left(\frac{1-\beta-\delta \kappa}{\kappa_{x}}\right) \phi_{x}+\phi_{\pi} & >1 \\
2 \sigma(1+\beta)+(1+\beta+\delta \kappa) \phi_{x}+\left(\kappa_{x}-2 \delta \kappa \sigma\right) \phi_{\pi}+\kappa_{x} & >0
\end{aligned}
$$

Proof. See appendix A.

Condition (7) can be interpreted as a generalization of the long-run Taylor principle that guarantees both determinacy and E-stability in the baseline "New Keynesian" framework; see Woodford (2003) and BM (2002). The difference between the conventional long-run Taylor principle and this generalized version relies on $\kappa$, which measures the impact of the interest rate on the inflation rate through the cost channel. In the line of Woodford (2003), this generalized version of the long run Taylor principle has the following economic interpretation: each percentage point of permanently higher inflation implies a permanent change in the output gap of $(1-\beta-\delta \kappa) / \kappa_{x}$ percentage points. Under the baseline case $(\delta=0)$, it is clear that any increment in the steady-state inflation leads to a higher output gap whereas under the cost channel $(\delta=1)$, it may lead to a permanent reduction in the output gap. ${ }^{10}$ The left-handside of (7) determines the long run increase of the interest rate given by the Taylor rule for each unit of increment in the steady-state inflation rate. Note that under the cost channel the traditional Taylor principle, $\phi_{\pi}>1$, may not longer imply its long run version as in the baseline case. This implication is less likely to occur if either the cost channel or the reaction to the output gap in the Taylor rule is weak. For example, in the case in which the Taylor rule does not respond to the output gap, i.e. $\phi_{x}=0$, condition (7) collapses to the traditional Taylor principle.

\footnotetext{
${ }^{9}$ For details see Blanchard and Kahn (1980).

${ }^{10}$ The output gap increases in the long run if $1-\beta>\kappa$.
} 
Inequality (8) is a second necessary condition for determinacy. In the baseline "New Keynesian" model, such condition is redundant and hence does not impose any constraint on the policy parameters. Nevertheless, when the cost channel is active, condition (8) may impose additional restrictions for determinacy. Assuming $\delta=1$ and replacing $\kappa_{x}$ in the term $\kappa_{x}-2 \delta \kappa \sigma$, it is straightforward to note that condition (8) binds if the inverse of the intertemporal elasticity of the substitution is greater than the inverse of the elasticity of the labor supply, $\eta<\sigma$. The previous implication is important because even in the case in which the central bank does not respond to the output gap, the traditional Taylor principle will not longer guarantee determinacy. To see this, note that if $\phi_{x}=0, \phi_{\pi}$ is two-sided constrained and therefore the traditional Taylor principle is not a sufficient condition for determinacy.

$$
1<\phi_{\pi}<\frac{2 \sigma(1+\beta)+\kappa(\eta+\sigma)}{\kappa(\sigma-\eta)}
$$

The economic intuition goes as follows: if the central bank's aggressiveness against inflation is too high, firms will raise prices even for a negative output gap, when the cost channel is active. In that case, the cost channel effect dominates the reduction of real wages (due to lower output) in such a way that high enough interest rates can generate self-fulfilling inflation expectations. Note that, the higher is the labor supply elasticity (i.e. the lower $\eta$ ), the lower is the reduction of real wages in response to a lower output. Moreover, the lower is the intertemporal elasticity of substitution (i.e. the higher is $\sigma$ ), the weaker is the effect of the interest rate (through the traditional demand channel) on the output gap and consequently on real wages and inflation. Under both cases, the cost channel offsets the effect of a negative output gap and hence indeterminacy follows.

To study the stability of REE under adaptive learning, we follow Evans and Honkapohja (2001, chapter 10) and assume that agents utilize a perceived law of motion (PLM) for $y_{t}$ that corresponds to the minimal state variable (MSV) solution (see McCallum 1983) to the system (5). The PLM can be written as:

$$
y_{t}=a+c \mu_{t}
$$

Using this PLM, agents form expectations of $y_{t+1}$ :

$$
E_{t} y_{t+1}=a+c \rho \mu_{t}
$$

Plugging these expectations into (5) delivers a T-mapping from the PLM to the actual law of motion (ALM):

$$
y_{t}=T_{a}(a)+T_{c}(c) \mu_{t}
$$


The rational expectations solution consists of values such that $\bar{a}=T_{\bar{a}}(\bar{a})$ and $\bar{c}=T_{\bar{c}}(\bar{c})$. The answer of the question of whether the system (5) is stable under learning is given by the principle of E-stability, which comes from analyzing the local asymptotic stability of the following matrix differential equation

$$
\frac{\partial T(a, c)}{\partial \tau}=T(a, c)-(a, c)
$$

evaluated at the REE solution $(\bar{a}, \bar{c})$. Specifically, the REE solution of the system (5) is E-stable or learnable if all real parts of the eigenvalues of

$$
\begin{aligned}
D T_{a}(\bar{a}) & =\Omega \\
D T_{c}(\bar{c}) & =\rho^{\prime} \otimes \Omega
\end{aligned}
$$

are lower than 1 .

McCallum (2007) shows that determinacy is a sufficient (though not necessary) condition for E-stability for a broad class of models, including the one in this paper. Hence, the generalized long run Taylor principle and condition (8) are sufficient for E-stability, i.e. all RE solution of (5) has the property of E-stability. Yet, we further need to check whether an indeterminate equilibria are E-stable or not. Propositions 2 summarizes the necessary and sufficient conditions for E-stability.

Proposition 2. Under contemporaneous data interest rate rules, the necessary and sufficient condition for an $M S V$ solution $(0, \bar{c})$ to be E-stable is that

$$
\left(\frac{1-\beta-\delta \kappa}{\kappa_{x}}\right) \phi_{x}+\phi_{\pi}>1
$$

Proof. See appendix B.

Condition for E-stability given in Proposition 2 is identical to the generalized long run Taylor principle defined above. Therefore, determinacy is sufficient for E-stability. Yet, if condition for determinacy (8) is not redundant, i.e. $\eta<\sigma$, indeterminate equilibria may be learnable. In order to gain an insight into the effects of the cost channel and the alternative policy rules specifications on determinacy and learnability conditions, we illustrate the results by using a calibrated case. Table 1 summarizes the benchmark parameterization. 

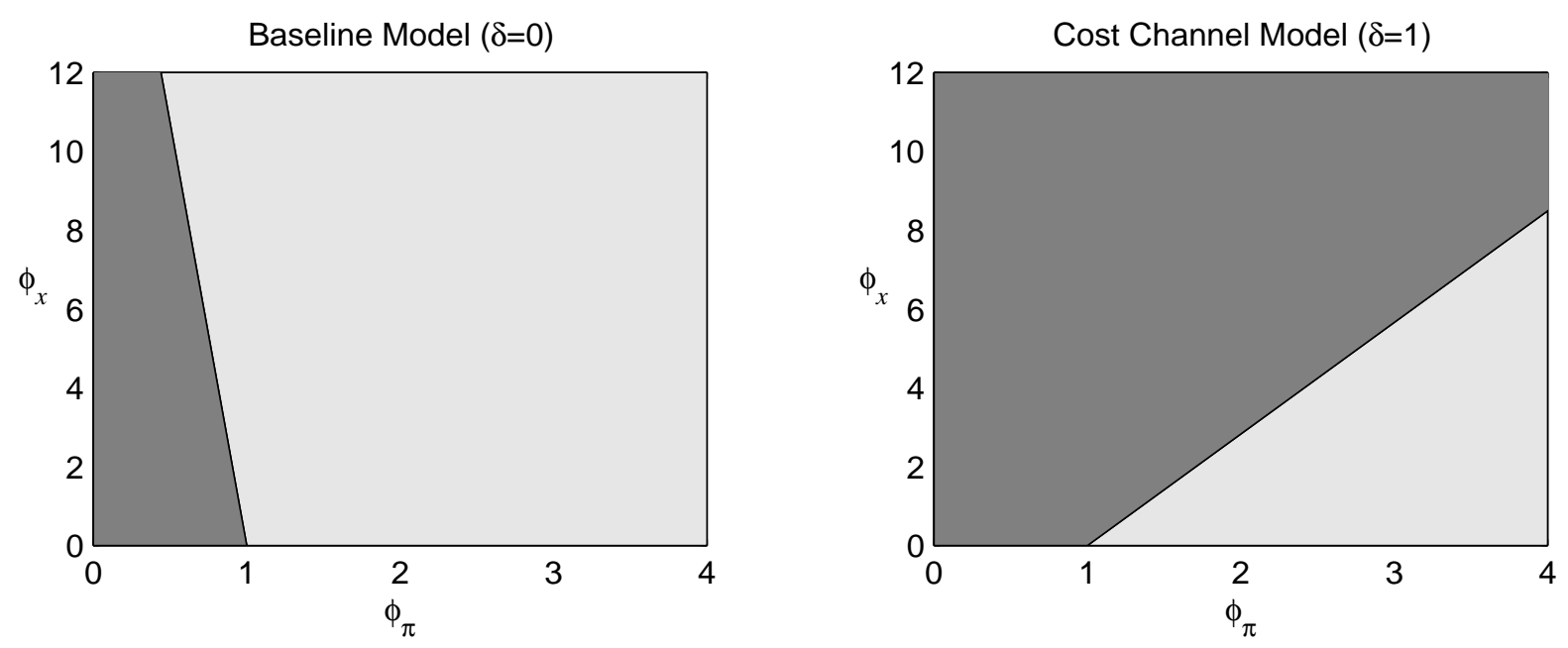

Determinate and E-stable

Indeterminate and E-unstable

Figure 1: Regions of determinacy and expectational stability for contemporaneous data policy rules. Left panel corresponds to the baseline model $(\delta=0)$. Right panel corresponds to the cost channel model $(\delta=1)$.

Table 1: Baseline Parameterization

\begin{tabular}{llr}
\hline \hline$\delta$ & Dichotomous parameter for the cost channel & 0 or 1 \\
$\theta$ & Probability of not adjusting prices & 0.75 \\
$\beta$ & Discount factor & 0.99 \\
$\sigma$ & Coefficient of risk aversion & 1.5 \\
$\eta$ & Inverse of the elasticity of labor supply & 1 \\
$\phi_{\pi}$ & Reaction to inflation & $0 \leq \phi_{\pi} \leq 12$ \\
$\phi_{x}$ & Reaction to output gap & $0 \leq \phi_{x} \leq 4$ \\
\hline \hline
\end{tabular}

Parameters $\eta$ and $\sigma$ are taken from RW (2006). We let $\delta$ to take two possible values: 0 or 1 , where the former characterizes baseline model, whereas the latter characterizes the model with the cost channel. As it is common in the literature on the Calvo (1983) pricing technology, we let the probability of not adjusting prices, $\theta=0.75$. We set $\beta$ to be equal to 0.99 , which implies an annualized of real interest rate of $4 \%$. As in BM (2002) we calibrate the policy reaction parameters for non-negative values.

Figure (1) depicts determinacy and E-stable regions as functions of both $\phi_{\pi}$ and $\phi_{x}$, with the rest of the parameters set at their baseline values. The figure of the left side depicts the baseline case $(\delta=0)$ whereas the figure of the right shows the cost channel case $(\delta=1)$. The main 
effect of the cost channel is to rotate the line describing the border between the determinate and E-stable region and indeterminate and E-unstable region. That border is given by the generalized long-run Taylor principle (equations 7 and 9). Under the cost channel, the set of parameter values in the policy rule that are consistent with determinacy and learnability are a subset of those for the baseline model. This is because $\kappa$, which measures the effect of the cost channel, alters long-run Taylor principle implied by the "new keynesian" case. In the calibrated case the slope of the long-run Taylor principle switches from negative to positive since $1-\beta<\kappa$ (the same holds under alternative calibrations considered in Table 2 below).

Three implications arise when the cost channel is active. First, in contrast to the baseline model, the traditional Taylor principle, $\phi_{\pi}>1$, does not always imply its long-run version and hence, (if $\phi_{x}>0$ ) such policy may not be sufficient for both determinacy and E-stability. Second, a passive policy reaction to inflation, i.e. $\phi_{\pi}<1$, never generates determinacy and Estability. Third, since in the parameterization $\eta<\sigma$, another implication of the cost channel is that the reaction to inflation has a upper bound for determinacy. Thus, provided a null response to the output gap, $\phi_{\pi}$ must lie between 1 and 144 (not shown in the graph) to guarantee a determinate equilibrium while in the baseline model a $\phi_{\pi}$ bigger than 1 is sufficient for determinacy.

\subsubsection{Forward data in the Taylor rule}

"Forward expectations" Taylor rules adopt the following form

$$
i_{t}=\phi_{\pi} E_{t} \pi_{t+1}+\phi_{x} E_{t} x_{t+1}
$$

where $\phi_{\pi}$ and $\phi_{x}$ are non-negative. We reduce the system of equations (1), (2) and (10) to two equations involving the endogenous variables $x_{t}$ and $\pi_{t}$. The reduced system takes the form of (5), where $\Omega$ is defined by

$$
\Omega=\frac{1}{\sigma}\left[\begin{array}{cc}
\delta \kappa \sigma \phi_{\pi}-\kappa_{x}\left(\phi_{\pi}-1\right)+\sigma \beta & \kappa_{x} \sigma-\kappa_{x} \phi_{x}+\delta \kappa \sigma \phi_{x} \\
-\left(\phi_{\pi}-1\right) & \sigma-\phi_{x}
\end{array}\right]
$$

The following proposition summarizes the necessary and sufficient conditions for a rational expectations equilibrium to be determinate.

Proposition 3. Under interest rate rules with forward expectations the necessary and sufficient 
conditions for determinacy are that

$$
\begin{aligned}
(\beta+\delta \kappa) \phi_{x}-\delta \kappa \sigma \phi_{\pi} & <\sigma(1+\beta) \\
\delta \kappa \sigma \phi_{\pi}-(\beta+\delta \kappa) \phi_{x} & <\sigma(1-\beta) \\
(1+\beta+\delta \kappa) \phi_{x}+\left(\kappa_{x}-2 \delta \kappa \sigma\right) \phi_{\pi} & <2 \sigma(1+\beta)+\kappa_{x} \\
\left(\frac{1-\beta-\delta \kappa}{\kappa_{x}}\right) \phi_{x}+\phi_{\pi} & >1
\end{aligned}
$$

Proof. See appendix C.

The analysis of E-stability is analogous to section 3.1.1 above. The following proposition provides the conditions for E-stability of the MSV solution.

Proposition 4. Under interest rate rules with forward expectations, the necessary and suffcient conditions for an $M S V$ solution $(0, \bar{c})$ to be E-stable are that

$$
\begin{aligned}
\delta \kappa \sigma \phi_{\pi}-(\beta+\delta \kappa) \phi_{x} & <\sigma(1-\beta) \\
\left(\frac{1-\beta-\delta \kappa}{\kappa_{x}}\right) \phi_{x}+\phi_{\pi} & >1
\end{aligned}
$$

Proof. See appendix D.

Propositions 3 and 4 show that the cost channel alters the conditions for both determinacy and learnability relative to the baseline model. Again, the generalized long-run Taylor principle is a necessary condition for determinacy and E stability and thus the same implications discussed in section 3.1.1 apply. Yet, a Taylor rule with forward expectations requires additional conditions not only for determinacy but also for E stability ${ }^{11}$. For example, to the extent that $\phi_{x}=0$, conditions (13) and (16) imply the following inequality

$$
\phi_{\pi}<\frac{(1-\beta)}{\delta \kappa}
$$

whereas conditions (15) and (17) imply the traditional Taylor principle

$$
\phi_{\pi}>1
$$

Notice that under the baseline case $(\delta=0)$, the first inequality (18) goes to infinite and hence

\footnotetext{
${ }^{11}$ Note that condition (13) never binds if $\delta=0$. Hence, under the baseline "New Keynesian" model the long-run Taylor principle is necessary and sufficient for E-stability as BM showed. Yet, this does not hold when the cost channel is active.
} 

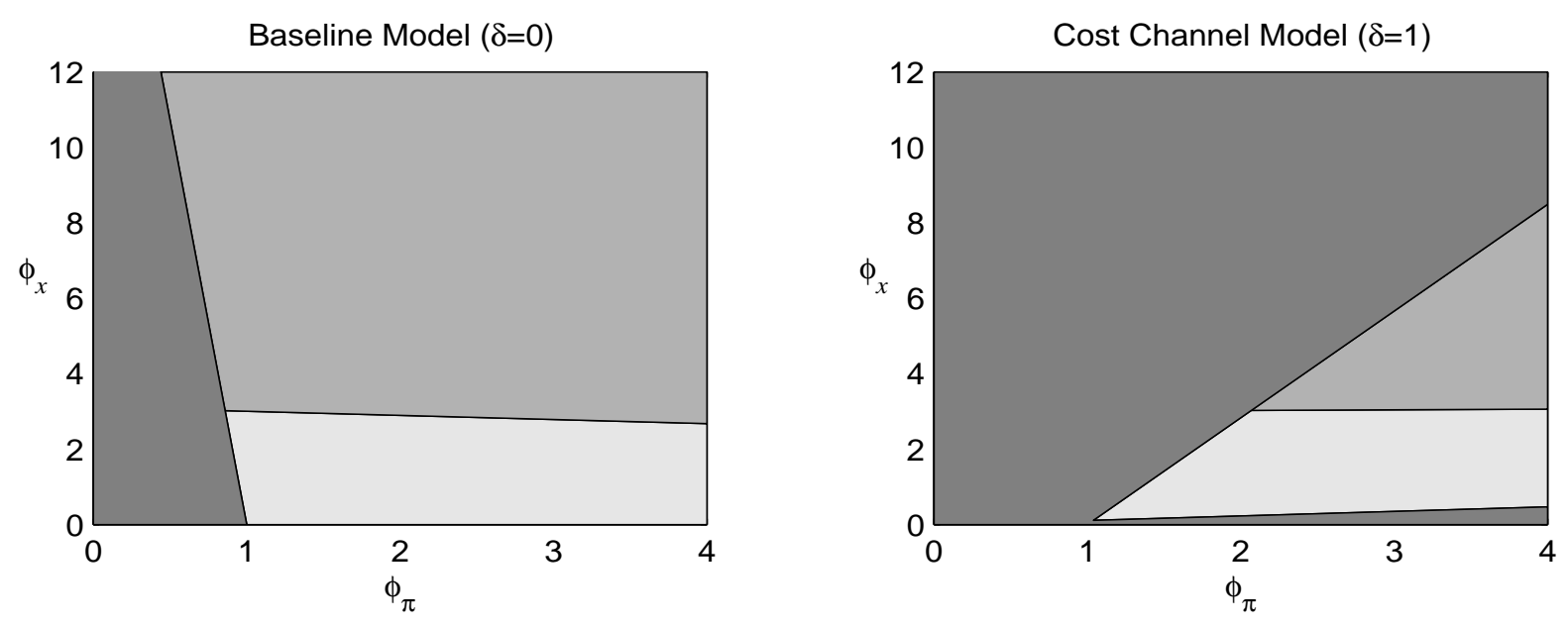

Determinate and E-stable

Indeterminate and E-stable

Indeterminate and E-unstable

Figure 2: Regions of determinacy and expectational stability for forward expectations policy rules. Left panel corresponds to the baseline model $(\delta=0)$. Right panel corresponds to the cost channel model $(\delta=1)$.

the traditional Taylor principle is a necessary condition for both determinacy and E-stability. ${ }^{12}$ In contrast, under the cost channel $(\delta=1)$, the limit given by (18) can be higher or lower than 1. If the limit (18) is below 1 , i.e. $1-\beta<\kappa$, and the policy reaction to the output gap is zero (i.e. $\phi_{x}=0$ ), determinacy and E-stability are never attainable. ${ }^{13}$ The latter is a remarkable result since the idea that the Taylor principle or "active" policy leads to determinacy and stability under learning is a celebrated result in the literature.

To illustrate these findings, figure (2) plots the intersections of the regions of determinacy and learnability. The figure of the left side depicts the baseline case $(\delta=0)$ whereas the figure of the right shows the cost channel case $(\delta=1)$. The figure of the left indicates that a forecastbased Taylor rule described by $\phi_{\pi}>1$ and a relatively small response to output gap guarantees a determinate and learnable equilibrium. Moreover, a passive reaction to inflation may also promote stability if it is accompanied by a sufficient reaction to the output gap. Contrary to this baseline case, when the cost channel is active the traditional Taylor Principle (i.e. $\phi_{\pi}>1$ ) does not guarantee a determinate and E-stable equilibrium even if $\phi_{x}$ is zero ${ }^{14}$. Overall, the

\footnotetext{
${ }^{12}$ As emphasized by BM (2002), under forward expectations specification $\phi_{\pi}$ has an upper bound given by condition (14). Hence, the traditional Taylor principle is a necessary but not sufficient condition for determinacy. Yet, the traditional Taylor principle is necessary and sufficient for E-stability in the model without cost channel.

${ }^{13}$ Notice that if $1-\beta<\kappa$, the long run Taylor principle is not implied by its traditional version; see section 3.1.1 for a detailed discussion.

${ }^{14}$ This result holds for the alternative calibrations see Table 2.
} 
area of determinacy and E- stability that induce determinacy and learnability shrinks notably.

A key question is why indeterminacy is more likely under the cost channel. As was discussed in the case of contemporaneous rules, indeterminacy under the cost channel arises because the supply-side effect of the interest rate, namely the cost channel, outweighs the demand-side effect. This implies a narrower range for the reaction to inflation in the policy rule, but still an active policy may guarantee a determinate equilibrium (as long as the inflation reaction is not too high and the reaction to output gap is nil or moderate). Yet, under forward-looking rules, indeterminacy emerges if the interest rate responds more than one-for-one to inflation expectations and it does not respond to output fluctuations. The economic intuition is that a rise in the interest rate (due to inflationary expectations) induces an increase in the current real interest rate, which not only reduces the current output gap and real wages, but also rises future output gap and hence renders inflation expectations to be self-fulfilling. ${ }^{15}$ A modest reaction to output gap expectations may dampen output gap expectations and therefore may alleviate the problem of indeterminacy (and instability under learning).

\subsection{Target Rules}

In this section we study those rules that can be implemented optimally under both discretion and commitment as in EH $(2003,2006)$. For each case, we study two forms of implementing an optimal rule through a linear reaction function for the interest rate. The first form is called "fundamental based" rule and is derived under the assumption of rational expectations. The second form is called "expectations-based" rule and is derived under the assumption that agents do not posses (initially) rational expectations.

\subsubsection{Discretionary Policy}

We now take the standard formulation of the central bank's loss function similar to the one derived in RW from first principles

$$
L_{0}=-(1 / 2) E_{0} \sum_{i=0}^{i=\infty} \beta^{i}\left[\lambda x_{t+i}^{2}+\pi_{t+i}^{2}\right]
$$

where $\lambda$ is the relative weight of output deviations. Following EH (2003), we treat $\lambda$ as free positive parameter. Yet, RW derive a microfounded optimal $\lambda$ as a function of deep parameters. Hence, we also analyze the case in which $\lambda$ is optimal which is give by $\lambda^{R W} \equiv \kappa\left(\frac{\eta+\sigma}{\theta}\right)$.

\footnotetext{
${ }^{15}$ Higher output gap in the next period arises as a consequence of two factors. First, higher consumption due to the intertemporal substitution. Second, lower production due to the cost channel.
} 
Optimal monetary policy under discretion implies to minimize (20) subject to versions of (1) and (2) equations modified to take into account the central bank's lack of commitment. It is straightforward to obtain the optimal condition that shows the trade-off between stabilizing domestic inflation and output gap, which reads:

$$
\pi_{t}=-\frac{\lambda}{\kappa_{o}} x_{t}
$$

where $\kappa_{o} \equiv \kappa_{x}-\delta \kappa \sigma \equiv \kappa[\eta-(\delta-1) \sigma]$. Note that when $\delta=0, \kappa_{o}=\kappa_{x}=\kappa(\eta+\sigma)$ and we get back to the standard trade-off found in Clarida et. al. (2000, hereafter CGG), whereas when $\delta=1$ we have that $\kappa_{o}=\kappa \eta$. Note also that the cost channel entails larger volatility of inflation since $\kappa(\eta+\sigma)>\kappa \eta$ (see RW 2006 for further details).

Fundamental-based reaction function EH (2003) discussed several forms of implementing the optimal plan given by (21). A first form is called "fundamental based" rule and implies that the central bank assumes that private agents have perfectly RE and that the REE takes the form of the MSV solution. Under such assumptions, the "fundamental based" rule for the interest rate reacts only to fundamental shocks. ${ }^{16}$

$$
i_{t}=\phi_{\mu} \mu_{t}
$$

We reduce the system of equations (1), (2) and (22) involving the endogenous variables $x_{t}$ and $\pi_{t}$. The reduced system takes the form of (5), where $\Omega$ is defined by

$$
\Omega=\left[\begin{array}{cc}
\beta+\frac{1}{\sigma} \kappa_{x} & \kappa_{x} \\
\frac{1}{\sigma} & 1
\end{array}\right]
$$

Note that the matrix (23) is independent from $\delta$ and it is exactly the same matrix analyzed by EH (2003). Thus, irrespective of whether the cost channel is present or not, the "fundamentalbased" optimal interest rate rule always leads to indeterminacy and instability under learning. In fact, EH (2003) prove that any linear policy rule of the form of (22) induces both indeterminacy and E-instability.

Expectations-based reaction function EH (2003) propose a second form, referred as "expectations-based" rule, which is derived under the assumption that agents do not posses $\mathrm{RE}$ and that their expectations can be observed by the central bank. The "expectations-based"

\footnotetext{
${ }^{16} \mathrm{EH}$ (2003) consider a more general case in which fiscal shocks appear in the fundamental-based reaction function. It is straightforward to show that the result of this section applies to the general case.
} 
optimal rule under discretion is obtained by solving $i_{t}$ from the structural equations (1) and (2) and the optimal condition (21)

$$
i_{t}=\phi_{\pi} E_{t} \pi_{t+1}+\phi_{x} E_{t} x_{t+1}+\phi_{\mu} \mu_{t}
$$

where the coefficients are

$$
\begin{aligned}
\phi_{\pi} & =\frac{\left(\lambda+\sigma \kappa_{o} \beta+\kappa_{o} \kappa_{x}\right)}{\left(\lambda+\kappa_{o}^{2}\right)} \\
\phi_{x} & =\frac{\sigma\left(\lambda+\kappa_{o} \kappa_{x}\right)}{\left(\lambda+\kappa_{o}^{2}\right)} \\
\phi_{\mu} & =\frac{\left(\sigma \kappa_{o}\right)}{\left(\lambda+\kappa_{o}^{2}\right)}
\end{aligned}
$$

Notice that when $\delta=0$ the "expectations-based" rule collapses to the one proposed by $\mathrm{EH}(2003)$.

$$
\begin{aligned}
\phi_{\pi}^{s} & =\frac{\left(\lambda+\sigma \kappa_{x} \beta+\kappa_{x}^{2}\right)}{\left(\lambda+\kappa_{x}^{2}\right)} \\
\phi_{x}^{s} & =\sigma \\
\phi_{\mu}^{s} & =\frac{\sigma \kappa_{x}}{\left(\lambda+\kappa_{x}^{2}\right)}
\end{aligned}
$$

where $s$ refers to the "standard" case.

We highlight that, regardless of whether the cost channel is active or not, the traditional Taylor principle holds under the "expectations-based" rule. That is, not only $\phi_{\pi}^{s}>1$ as EH (2003) point out, but also $\phi_{\pi}>1$ (the latter derives from $\kappa_{o}<\kappa_{x}$ ). Besides, numerical results show the optimal reaction under the cost channel model $\left(\phi_{\pi}\right)$ is lower than the one under the standard case $\left(\phi_{\pi}^{s}\right) .{ }^{17}$ In addition, given that $\kappa_{o}<\kappa_{x}$, the optimal reaction to output gap expectations under the cost channel is bigger than the one under the standard case.

The reduce form of the model under (24) takes the form of (5), where $\Omega$ is defined by

$$
\Omega=\psi\left[\begin{array}{cc}
(\beta+\kappa \delta) \lambda & \delta \sigma \lambda \kappa \\
-\kappa_{o}(\beta+\delta \kappa) & -\delta \sigma \kappa \kappa_{O}
\end{array}\right]
$$

with $\psi=\left(\lambda+\kappa_{o} \kappa_{x}-\delta \kappa \sigma \kappa_{o}\right)^{-1}$.

The system is determinate if and only if matrix $\Omega$ has both eigenvalues inside the unit circle and E-stable if all eigenvalues of $\Omega-I$ have negative real parts. One of the roots of $\Omega$

\footnotetext{
${ }^{17}$ The same result hold for different calibrations and different values of $\lambda$. The results are available upon request.
} 
is zero and the other one is given by

$$
r=\frac{\lambda(\beta+\kappa \delta)-\kappa \sigma \delta \kappa_{o}}{\lambda+\kappa_{o} \kappa_{x}-\kappa \sigma \delta \kappa_{o}}
$$

Note that under the standard case, i.e. $\delta=0, r$ is positive and lower that 1 , thus determinacy and E-stability immediately follows (see EH 2003). Under the cost channel, however, we found that only a subset of the parameter space is related to either determinacy or E-stability (see propositions 5 and 6). Therefore, the "expectations-based" optimal rule does not always lead to determinacy and learnability for all parameter values as in the standard "new keynesian" case.

Proposition 5. Under the expectations-based optimal rule derived under discretion the necessary and sufficient condition for a rational expectations equilibrium to be determinate is that

$$
\frac{\kappa^{2} \eta(\sigma-\eta)}{(\beta+\kappa+1)}<\lambda<\frac{\kappa^{2} \eta(\eta+\sigma)}{(\beta+\kappa-1)}
$$

Proof. See appendix E.

Proposition 6. Under the expectations-based optimal rule derived under discretion the necessary and sufficient condition for a MSV solution $(0, \bar{c})$ to be E-stable is that

$$
\lambda<\frac{\kappa^{2} \eta(\eta+\sigma)}{(\beta+\kappa-1)}
$$

Proof. See appendix F.

Using our baseline calibration, Propositions 5 and 6 imply that the "expectations-based" optimal rule leads to a determinate REE as long as $\lambda$ ranges between 0.05 and 0.24 and Estability follows if $\lambda$ is lower than 0.24 . For robustness, we also evaluate this finding under alternative parameterizations as in Woodford (1999, W), CGG (2000) and McCallum and Nelson $(1999, \mathrm{MN})$. Table 2 below summarizes the alternative parameterizations. Note that under Woodford's parameterization the slope coefficient of the Phillips curve is ten times smaller than the ones of CGG and MN (0.024 compared to 0.3), hence the effect of the output gap over inflation through the Phillips curve will be significantly smaller and the cost channel effect will become relative more important. Intuitively this explains why the permissible parameter range for $\lambda$ to guarantee both determinacy and E-stability shrinks under Woodford (1999)'s calibration. We also ask whether the determinacy and E-stability are attainable if the central bank uses the optimal $\lambda^{R W}$. Table 2 shows that only under Woodford's parameterization, $\lambda^{R W}$ 
lies inside the ranges for determinacy and E-stability. Interestingly, these results imply that the cost channel imposes tight restrictions even for optimal policy.

Table 2: Policy restrictions under discretion - "expectations-based"

\begin{tabular}{ccccc}
\hline \hline & Baseline & W (1999) & CGG (2000) & MN (1999) \\
\hline$\sigma$ & 1.5 & 0.157 & 1 & $1 / 0.164$ \\
$\kappa_{x}$ & 0.21 & 0.024 & 0.3 & 0.3 \\
Determinacy & $0.05<\lambda<0.24$ & $\lambda<0.05$ & $\lambda<0.32$ & $0.28<\lambda<0.39$ \\
E-stability & $\lambda<0.24$ & $\lambda<0.05$ & $\lambda<0.32$ & $\lambda<0.39$ \\
Optimal $\lambda^{R W}$ & 0.29 & 0.03 & 0.40 & 0.40 \\
\hline \hline
\end{tabular}

EH (2003) provide an intuition about why the "expectations-based" reaction function always leads to stability under both determinacy and learning. They argue that under such policy rule the traditional Taylor principle always holds, i.e. $\phi_{\pi}>1$, and thus, the central bank succeeds in stabilizing the economy towards the optimal REE. In contrast, in a model in which monetary policy works also through the cost channel this is no longer true. As we showed earlier, the traditional Taylor principle does not guarantee determinacy and learnability of the forward data Taylor rule (10), which indeed it has the same form of the "expectations-based" rule (24). Furthermore, under the forward data Taylor rule, the traditional Taylor principle does not imply its long-run version and consequently an active reaction against inflation expectations is not sufficient for determinacy and E-stability. As a consequence, when the cost channel matters and under discretionary policy, there are some parameter values under which the economy displays indeterminacy and/or expectational instability.

A key question is which conditions of Proposition 3 and 4 do not hold under the "expectations - based" rule and hence generate the indeterminacy and E-instability result. Figure (3) plots the determinacy and E-stable regions of the Taylor rule with forward data (10) as in figure (2). The dotted lines correspond to the rays $\left\{\phi_{\pi}^{s}, \phi_{x}^{s}\right\}$ and $\left\{\phi_{\pi}, \phi_{x}\right\}$ given by the "expectations-based" rule (24). The rays plot the combination of optimal parameters under different values of $\lambda$ (ranging from 0 to 1 ). Under the baseline model, the ray always stays in the determinate and E-stable area. Under the cost channel model, the numerical results show that as $\lambda$ increases, both $\phi_{\pi}$ and $\phi_{x}$ decrease and the ray crosses the long-run Taylor principle. We check this result by plugging the optimal parameters $\phi_{\pi}$ and $\phi_{x}$ into the long-run Taylor principle. The resulting expression collapses to the following condition, confirming that E-stability requires Proposition 5.

$$
\frac{\sigma\left(\kappa^{2} \eta(\eta+\sigma)-(\beta+\kappa-1) \lambda\right)}{\left(\lambda+\kappa^{2} \eta^{2}\right)}>0
$$



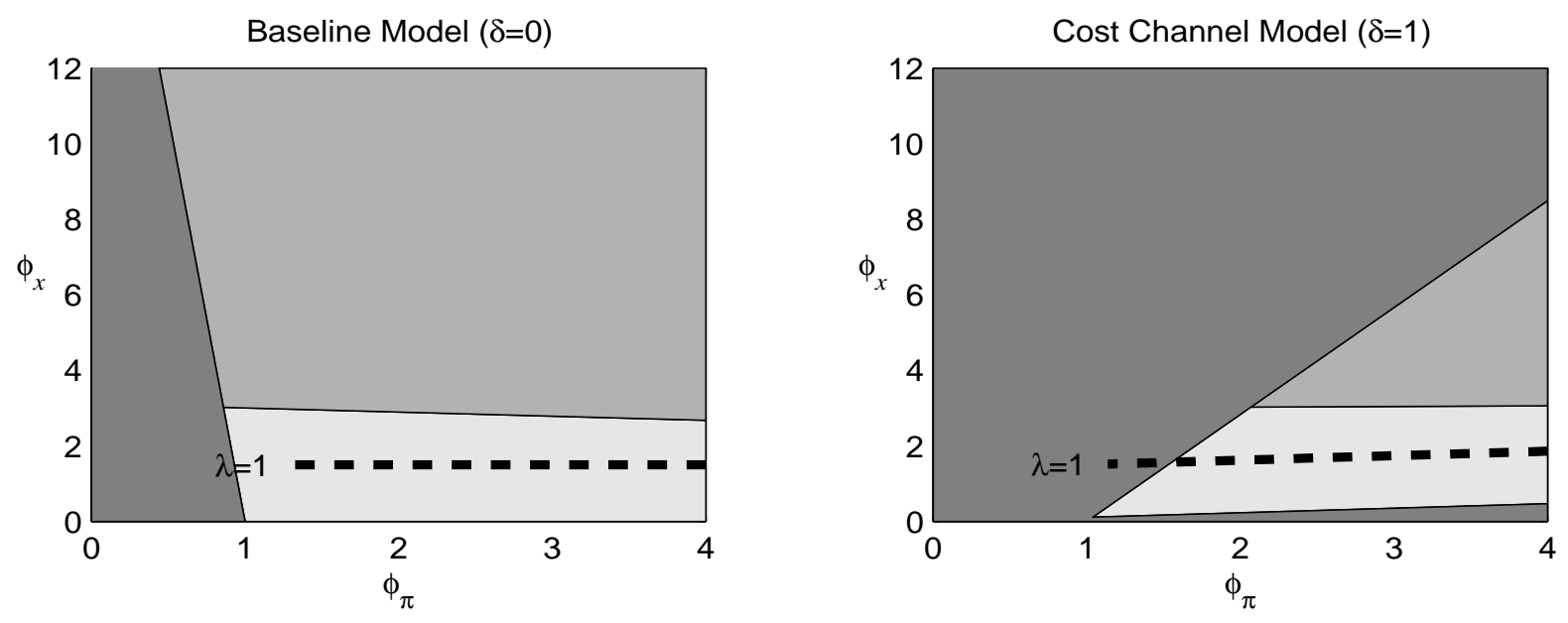

Determinate and E-stable

Indeterminate and E-stable

Indeterminate and E-unstable

Figure 3: Regions of determinacy and expectational stability for forward expectations policy rules. Left panel corresponds to the baseline model $(\delta=0)$. Right panel corresponds to the cost channel model $(\delta=1)$. The dotted lines plot the optimal parameters $\phi_{\pi}$ and $\phi_{x}$ under the "expectations-based" rule derived under discretion.

Another interesting implication of the previous analysis is that there exists a conflict between the desirable properties of an optimal discretionary rule in terms of the volatility that it entails and the learnability and determinacy criteria. Indeed, note that optimal condition (21) shows that the cost channel increases the trade-off between stabilizing inflation and the output gap $\left(\kappa_{0}<\kappa_{x}\right)$ and simultaneously it implies smaller optimal reaction to expected inflation with respect to the baseline model $\left(\phi_{\pi}<\phi_{\pi}^{s}\right)$. Yet, as shown in figure (3), the latter might induce undesirable properties in terms of both learnability and determinacy. Hence, the achievement of determinacy and learnability under the cost channel would imply a bigger reaction to inflation expectations - as the baseline model suggests $\left(\phi_{\pi}^{s}\right)$ - at the cost of larger macroeconomic volatility.

To sum up, our results suggest that EH's proposal to solve the instability of "fundamentalbased" rules by conditioning optimally on private sector expectations, can be misleading when the cost channel matters. As it was shown, the possible solution to this issue, as suggested by EH (2003), does not always provide stability under learning. As the next section will prove, this conclusion emerges also when the policymaker commits to an optimal policy rule. 


\subsubsection{Commitment Policy}

The policy problem is the following: Let's write the Lagrangian

$$
L_{0}=E_{0} \sum_{t=0}^{\infty} \beta^{t}\left\{\begin{array}{c}
\frac{1}{2}\left[\lambda x_{t}^{2}+\pi_{t}^{2}\right]+\varphi_{1, t}\left[x_{t}-x_{t+1}+\frac{1}{\sigma}\left(i_{t}-\pi_{t+1}\right)\right] \\
+\varphi_{2, t}\left[\pi_{t}-\beta \pi_{t+1}-\kappa_{x} x_{t}-\delta \kappa i_{t}\right]
\end{array}\right\}-\varphi_{1,0} \pi_{0}
$$

the first order conditions with respect to $\pi_{t}, x_{t}$ and $i_{t}$ are respectively:

$$
\begin{aligned}
\pi_{t}-\frac{1}{\sigma \beta} \varphi_{1, t-1}+\varphi_{2, t}-\varphi_{2, t-1} & =0 \\
\lambda x_{t}+\varphi_{1, t}-\frac{1}{\beta} \varphi_{1, t-1}-\kappa_{x} \varphi_{2, t} & =0 \\
\frac{1}{\sigma} \varphi_{1, t}-\delta \kappa \varphi_{2, t} & =0
\end{aligned}
$$

and $\pi_{0}=\bar{\pi}_{0}$.

Combining (27), (28), (29) we get the following set of equations ${ }^{18}$,

$$
\begin{aligned}
\varphi_{2, t} & =\left(1+\beta^{-1} \delta \kappa\right) \varphi_{2, t-1}-\pi_{t} \\
x_{t} & =\lambda^{-1} \beta^{-1} \sigma \delta \kappa \varphi_{2, t-1}+\lambda^{-1}\left(\kappa_{x}-\sigma \delta \kappa\right) \varphi_{2, t}
\end{aligned}
$$

Combining equations (1), (2) with (30) and (31), we can obtain the following reduced form,

$$
y_{t}=A+B E_{t} y_{t+1}+C y_{t-1}+D \mu_{t}
$$

where $y_{t}=\left[\pi_{t}, \varphi_{2, t}\right]^{\prime}, A=0$, and

$$
\begin{aligned}
& B=\vartheta\left[\begin{array}{cc}
\beta \lambda\left(\beta+\delta_{o}\right) & \sigma \beta \delta_{o} \kappa_{o} \\
-\beta \lambda\left(\beta+\delta_{o}\right) & -\sigma \beta \delta_{o} \kappa_{o}
\end{array}\right] \\
& C=\vartheta\left[\begin{array}{cc}
0 & \sigma \kappa_{o} \delta_{o}+\left(\sigma^{2} \delta_{o}^{2}+\beta \kappa_{o}^{2}\right)\left(\beta^{-1} \delta_{o}+1\right) \\
0 & \beta \lambda\left(\beta^{-1} \delta_{o}+1\right)-\sigma \kappa_{o} \delta_{o}
\end{array}\right] \\
& D=\vartheta\left[\begin{array}{c}
\lambda \beta \\
-\lambda \beta
\end{array}\right]
\end{aligned}
$$

with $\vartheta=\left(\beta \lambda+\sigma^{2} \delta_{o}^{2}+\beta \kappa_{o}^{2}\right)^{-1}, \delta_{o}=\delta \kappa$ and $\kappa_{o} \equiv \kappa_{x}-\delta \kappa \sigma$.

The MSV solution of (32) can be written as a function of the lagrange multiplier, $\varphi_{2, t-1}$,

\footnotetext{
${ }^{18}$ Note that different from the standard case, analyzed by EH (2006), we cannot eliminate the lagrange
} multipliers in order to get a tractable system. 
and the fundamental shock, $\mu_{t}$,

$$
\begin{aligned}
\pi_{t} & =b_{\pi} \varphi_{2, t-1}+c_{\pi} \mu_{t} \\
\varphi_{2, t} & =b_{\varphi} \varphi_{2, t-1}+c_{\varphi} \mu_{t}
\end{aligned}
$$

After replacing (33) and (34) (and their respective expected values) into (32), we obtain the following polynomial characterizing $b_{\varphi}$

$$
\beta b_{\varphi}^{2}-\gamma b_{\varphi}+1=0
$$

where $\gamma=\frac{\left(\sigma^{2} \delta_{o}^{2}+\beta \kappa_{o}^{2}+\lambda \beta\right)+\lambda\left(\beta+\delta_{o}\right)^{2}}{\left(\lambda\left(\beta+\delta_{o}\right)-\sigma \kappa_{o} \delta_{o}\right)}$. Unlike the standard model, both roots of (35) are not necessarily positive. To have positive roots we need $\gamma$ to be positive, which translates to the following condition $\lambda>\sigma \kappa^{2} \eta /(\beta+\kappa)$. Under such condition, the stable root of (35) is given by,

$$
\bar{b}_{\varphi}=(2 \beta)^{-1}\left[\gamma-\left(\gamma^{2}-4 \beta\right)^{1 / 2}\right]
$$

This root delivers a stationary REE, since $0<\bar{b}_{\varphi}<1$. The rest of coefficients are given:

$$
\begin{aligned}
& \bar{b}_{\pi}=\beta^{-1} \kappa \delta+\left(1-\bar{b}_{\varphi}\right) \\
& \bar{c}_{\pi}=-\bar{c}_{\varphi} \\
& \bar{c}_{\varphi}=-\beta \lambda\left[\left(\sigma^{2} \delta_{o}^{2}+\beta \kappa_{o}^{2}+\beta \lambda\right)+\sigma \kappa_{o} \delta_{o}\left(\delta_{o}+\beta\right)-\left(\lambda\left(\beta+\delta_{o}\right)-\sigma \kappa_{o} \delta_{o}\right) \beta\left(\rho-\bar{b}_{\pi}\right)\right]^{-1}
\end{aligned}
$$

We refer to this REE as the optimal REE. The next proposition summarizes this result.

Proposition 7. The optimal REE under a commitment policy is characterized by $0<\bar{b}_{\varphi}<1$ and $\bar{b}_{\pi}>0$ iff

$$
\frac{\sigma \kappa^{2} \eta}{(\beta+\kappa)}<\lambda
$$

Fundamental-based reaction function The "fundamental based" rule implies that the central bank assumes that private agents have perfectly RE and that the REE takes the form of the MSV solution. We replace the RRE solution of the form of (33) and (34), and their respective expectations into the structural relationships (1) and (2) - after using (31) . Then, we solve for $i_{t}$ and the resulting equation is called "fundamental-based" optimal rule,

$$
i_{t}=\phi_{\varphi} \varphi_{2, t-1}+\phi_{\mu} \mu_{t}
$$


where $\phi_{\varphi}$ and $\phi_{\mu}$ are given by

$$
\begin{gathered}
\phi_{\varphi}=(\beta \Delta)^{-1}\left(\begin{array}{c}
\bar{b}_{\pi}^{2} \beta^{2} \lambda^{2}-\delta_{o} \kappa_{x} \sigma^{2}\left(\bar{b}_{\varphi} \beta \kappa_{o}+\delta_{o} \sigma\right) \\
+\bar{b}_{\pi} \beta \lambda \sigma\left(\left(-1+\bar{b}_{\varphi}\right) \beta \kappa_{o}-\beta \delta_{o} \sigma+\delta_{o}\left(-\kappa_{x}+\sigma\right)\right)
\end{array}\right) \\
\phi_{\mu}=\Delta^{-1}\left(\begin{array}{c}
\beta \bar{c}_{\pi} \kappa_{o} \kappa_{x} \lambda \rho-\beta \kappa_{o}\left(-\bar{c}_{\varphi} \kappa_{o} \kappa_{x} \rho+\left(-1+\bar{b}_{\varphi}\right) \lambda\left(1-\bar{c}_{\pi}+\beta \bar{c}_{\pi} \rho\right)\right) \sigma \\
-\delta_{o} \lambda\left(1+\bar{c}_{\pi}(-1+\beta \rho) \sigma^{2}+\bar{b}_{\pi} \beta \lambda\left(\left(-1+\bar{c}_{\pi}\right) \lambda+\beta \bar{c}_{\varphi} \kappa_{o} \rho \sigma\right)\right)
\end{array}\right)
\end{gathered}
$$

with $\Delta=\lambda\left(\beta \kappa_{o} \kappa_{x}+\bar{b}_{\pi} \beta\left(\beta+\delta_{o}\right) \lambda+\left(-1+\bar{b}_{\varphi}\right) \beta \delta_{o} \kappa_{o} \sigma+\delta_{o}^{2} \sigma^{2}\right)$.

Combining the "fundamental-based" reaction function (36) with (1), (2) and (31) we collapse a system of the form of $(32)$, where $y_{t}=\left[\pi_{t}, \varphi_{2, t}\right]^{\prime}, \Gamma=0$, and

$$
\begin{aligned}
& \Omega=\psi\left[\begin{array}{cc}
\sigma^{-1}\left(\beta \kappa_{o}\left(\beta \sigma+\kappa_{x}\right)-\beta \sigma^{2} \delta_{o}\right) & \beta \kappa_{o}^{2} \kappa_{x} \lambda^{-1} \\
\beta \lambda \sigma^{-1} & \beta \kappa_{o}
\end{array}\right] \\
& \Phi=\psi\left[\begin{array}{cc}
0 & (\beta \lambda \sigma)^{-1}\left(\delta_{o} \sigma\left(\left(\beta \lambda \phi_{\varphi}+\sigma \kappa_{x}\right)\left(\beta \kappa_{o}-\sigma \delta_{o}\right)-\beta \kappa_{o} \kappa_{x} \sigma\right)-\beta^{2} \kappa_{o} \kappa_{x} \lambda \phi_{\varphi}\right) \\
0 & -\sigma^{-1}\left(\beta \lambda \phi_{\varphi}+\sigma^{2} \delta_{o}\right)
\end{array}\right]
\end{aligned}
$$

with $\psi=\left(\beta \kappa_{o}-\sigma \delta_{o}\right)^{-1}, \delta_{o} \equiv \delta \kappa$ and $\kappa_{o} \equiv \kappa_{x}-\delta \kappa \sigma$. The MSV solution can be written as $y_{t}=a+b y_{t-1}+c \mu_{t}$, where the REE is given by $(a, b, c)=(\bar{a}, \bar{b}, \bar{c})$.

Next, we analyze whether the "fundamental-based" optimal policy guarantees a learnable REE. Under the standard new Keynesian model, EH (2006) show that the fundamental reaction function leads to instability under learning. Remarkably, our results stand in contrast with those of $\mathrm{EH}(2006)$. In fact, the fundamental-based reaction function (36) can induce a E-stable under a specific parameterization. In particular, if the inverse of the intertemporal elasticity of substitution $(\sigma)$ is greater than the inverse of the intertemporal elasticity of labor supply $(\eta)$ the REE is E-stable.

Proposition 8. Under the fundamental-based reaction function derived under commitment, the necessary and sufficient condition for the optimal REE to be E-stable is that $\sigma>\beta \eta$

Proof. See appendix G.

Proposition 8 shows how in the model with the cost channel the strength of demand-side effects is crucial for E-stability. The intuition follows the analysis of contemporaneous rules. In that case, we emphasized that if $\eta<\sigma$ and the central bank's aggressiveness against inflation are too high, firms will raise prices even for a negative output gap because the cost channel dominates the reduction of real wages (due to lower output). Thus, high enough interest rate can generate self-fulfilling inflation expectations. In the case of the fundamental optimal rule under commitment such intuition applies to E-stability. 
Determinacy is evaluated numerically and for this case $\lambda$ is treated as a free policy parameter ranging from 0 to 1 . Numerical results reveal that for the benchmark calibration determinacy is guaranteed if $\lambda>0.05$ and the REE is always E-stable. We also check whether alternative calibrations yield determinacy and stability under learning; see Table 3 below. Furthermore, the optimal fundamental rule delivers both determinacy and learnability under most parameterizations. Thus, three out of four parameterizations guarantee determinacy and Estability under $\lambda^{R W}$. Woodford's parameterization is the only one that induces expectational instability and indeterminacy under the optimal $\lambda^{R W}$. The latter may be explained by the fact that in Woodford's parameterization the effect of the output gap over inflation through the Phillips curve will be significantly smaller than that of the cost channel.

Table 3: Policy restrictions under commitment - "fundamental-based"

\begin{tabular}{ccccc}
\hline \hline & Baseline & W (1999) & CGG (2000) & MN (1999) \\
Determinacy & $0.05<\lambda$ & $0.44<\lambda$ & $0.02<\lambda$ & $0.11<\lambda$ \\
E-stability & Yes & No & Yes & Yes \\
Optimal $\lambda^{R W}$ & 0.29 & 0.03 & 0.40 & 0.40 \\
\hline \hline
\end{tabular}

Note: Parameterizations are the same as in Table 2

The above results bring about some interesting implications. First, a commitment policy that assumes RE delivers determinacy and E-stability when monetary also works through the cost channel if and only if the strength of the demand channel exceeds that of the cost channel. Second, if an optimal $\lambda^{R W}$ is chosen, the fundamental based rule under commitment is more likely to generate both determinacy and E-stability than the expectations based rule under discretion. This last result indicates that the ability to commit may help in the alleviation of the instability problem when the cost channel is present without conditioning policy response to inflation expectations. Yet, this type of policy could be less appealing given the difficulties that the implementation of fundamental rules impose in practice.

Expectations-based reaction function Following EH (2006) we also study the so-called "expectations-based" optimal rule under commitment. After plugging (30) and (31) into the aggregate supply (1), we can replace $x_{t}$ for $\varphi_{2, t}$. Then, we express the aggregate demand (2) in terms of $\varphi_{2, t}$ by using equation (31). By solving $i_{t}$ from the resulting equations, we get the following expression for the "expected-based" optimal rule,

$$
i_{t}=\phi_{L} \varphi_{2, t-1}+\phi_{\pi} E_{t} \pi_{t+1}+\phi_{\varphi} E_{t} \varphi_{2, t+1}+\phi_{\mu} \mu_{t}
$$


where,

$$
\begin{aligned}
\phi_{L} & =-\frac{\delta_{o}\left(\kappa_{o}-\beta^{-1} \sigma \delta_{o}\right)^{-1} \beta^{-1} \sigma+\left(\kappa_{x} \lambda^{-1} \kappa_{o}+1\right)^{-1}\left(1+\beta^{-1} \delta_{o}+\kappa_{x} \lambda^{-1} \beta^{-1} \sigma \delta_{o}\right)}{\lambda\left(\kappa_{o}-\beta^{-1} \sigma \delta_{o}\right)^{-1} \sigma^{-1}-\delta_{o}\left(\kappa_{x} \lambda^{-1} \kappa_{o}+1\right)^{-1}} \\
\phi_{\pi} & =\frac{\left(\kappa_{x} \lambda^{-1} \kappa_{o}+1\right)^{-1} \beta+\lambda\left(\kappa_{o}-\beta^{-1} \sigma \delta_{o}\right)^{-1} \sigma^{-1}}{\lambda\left(\kappa_{o}-\beta^{-1} \sigma \delta_{o}\right)^{-1} \sigma^{-1}-\delta_{o}\left(\kappa_{x} \lambda^{-1} \kappa_{o}+1\right)^{-1}} \\
\phi_{\varphi} & =\frac{\left(\kappa_{o}-\beta^{-1} \sigma \delta_{o}\right)^{-1} \kappa_{o}}{\lambda\left(\kappa_{o}-\beta^{-1} \sigma \delta_{o}\right)^{-1} \sigma^{-1}-\delta_{o}\left(\kappa_{x} \lambda^{-1} \kappa_{o}+1\right)^{-1}} \\
\phi_{\mu} & =\frac{\left(\kappa_{x} \lambda^{-1} \kappa_{o}+1\right)^{-1}}{\lambda\left(\kappa_{o}-\beta^{-1} \sigma \delta_{o}\right)^{-1} \sigma^{-1}-\delta_{o}\left(\kappa_{x} \lambda^{-1} \kappa_{o}+1\right)^{-1}}
\end{aligned}
$$

The reduced form dynamics for the vector $y_{t}=\left[\pi_{t}, \varphi_{2, t}\right]^{\prime}$ under takes the form of (32), where $\Gamma=0$, and

$$
\Omega=\left[\begin{array}{cc}
\frac{\lambda\left(\phi_{\pi}-1\right)}{\sigma\left(\kappa_{o}-\beta^{-1} \sigma \delta_{o}\right)} & -\frac{\left(\kappa_{o}-\lambda \sigma^{-1} \phi_{\varphi}\right)}{\left(\kappa_{o}-\beta^{-1} \sigma \delta_{o}\right)} \\
-\frac{\left(\beta+\delta_{o} \phi_{\pi}\right)}{\left(\kappa_{x} \lambda^{-1} \kappa_{o}+1\right)} & -\frac{\delta_{o} \phi_{\varphi}}{\left(\kappa_{x} \lambda^{-1} \kappa_{o}+1\right)}
\end{array}\right] \Phi=\left[\begin{array}{cc}
0 & \frac{\left(\beta^{-1} \sigma \delta_{o}+\lambda \sigma^{-1} \phi_{L}\right)+\left(1+\beta^{-1} \delta_{o}\right)\left(\kappa_{o}-\beta^{-1} \sigma \delta_{o}\right)}{\left(\kappa_{o}-\beta^{-1} \sigma \delta_{o}\right)} \\
0 & \frac{\left(1+\beta^{-1} \delta_{o}+\kappa_{x} \lambda^{-1} \beta^{-1} \sigma \delta_{o}-\delta_{o} \phi_{L}\right)}{\left(\kappa_{x} \lambda^{-1} \kappa_{o}+1\right)}
\end{array}\right]
$$

The MSV solution can be written as $y_{t}=a+b y_{t-1}+c \mu_{t}$, were the REE is given by $(a, b, c)=$ $(\bar{a}, \bar{b}, \bar{c})$. In appendix $\mathrm{H}$ it is shown that a necessary condition for determinacy is the same to the one characterizing the optimal REE (see proposition 7).

Proposition 9. Under the expected-based reaction function derived under commitment, the necessary condition for a rational expectations equilibrium to be determinate is that

$$
\frac{\sigma \kappa^{2} \eta}{(\beta+\kappa)}<\lambda
$$

Proof. See appendix H.

In the standard sticky price model, EH (2006) prove that the "expectations-based" reaction function guarantees stability under learning for all parameter configurations. In contrast, when the economy features the cost channel, the "expectations-based" policy rule given by (37), may or may not guarantee E-stability of the optimal REE.

Proposition 10. Under the expected-based reaction function derived under commitment, the necessary and sufficient condition for the optimal REE $(0, \bar{b}, \bar{c})$ to be E-stable is that

$$
\frac{\kappa \eta \sigma}{\beta+\kappa}<\lambda<\frac{\kappa^{2} \eta(\eta+\sigma)}{(\beta+\kappa-1)}
$$


Proof. See appendix I.

We evaluate numerically the thresholds given in Proposition 9 and 10 (see Table 4 below). In the case of E-stability, W(1999)'s calibration has yielded a tighter space for $\lambda$ compared to the rest. This is because in Woodford's parameterization the effect of the output gap over inflation through the aggregate supply is significantly smaller than the cost channel effect and therefore it is harder to induce E-stability. Yet, W(1999)'s calibration is the only one in which both determinacy and E-stability are always guarantee if an optimal $\lambda^{R W}$ is chosen. The bottom line is that EH's proposal to the resolution of the instability does not always work even for commitment policies when the cost channel matters.

Table 4: Policy restrictions under commitment - "expectational-based"

\begin{tabular}{ccccc}
\hline \hline & Baseline & W (1999) & CGG (2000) & MN (1999) \\
Determinacy & $0.01<\lambda$ & $0<\lambda$ & $0.02<\lambda$ & $0.11<\lambda$ \\
E-stability & $0.12<\lambda<0.24$ & $0<\lambda<0.05$ & $0.13<\lambda<0.32$ & $0.25<\lambda<0.39$ \\
Optimal $\lambda^{R W}$ & 0.29 & 0.03 & 0.40 & 0.40 \\
\hline \hline
\end{tabular}

Note: Parameterizations are the same as in Table 2

We summarize our main results under target rules as follows. First, when the cost channel matters, conditioning policy on private sector expectations threatens E-stability under both discretion and commitment. Second, a policy that it is a source of instability under learning without the cost channel, i.e. "fundamental rule" under commitment, is a possible antidote when the cost channel is active. The "fundamental rule" under commitment (32) considers a reaction to a lag multiplier which captures the importance of history or lag endogenous variables under commitment. Hence, this findding suggests that problems of instability under learning when the cost channel is active might be mitigated by endowing instrument rules with interest rate smoothing as Bullard and Mitra (2006) pointed out.

\section{Conclusions}

In this paper we have studied determinacy and E-stability of different monetary policy rules when the cost channel matters. Particularly, we have extended BM (2002) and EH (2003, 2006) analyses in the presence of supply side effects of monetary policy through the cost channel. Our results show that the cost channel modifies the standard conditions for both determinacy and learnability when the central banks operates with either instrument or target rules. Remarkably, the traditional Taylor principle does not implies its long-run version in a model with the cost channel. 
In general, the presence of the cost channel threatens the determinacy and learnability of the rational expectations equilibrium. Moreover, popular policies to counteract instability, like the traditional Taylor principle or "expectations-based" reaction functions, may not be effective or could even be counterproductive. Interestingly, a policy that it is a source of instability under learning without the cost channel, i.e. "fundamental rule" under commitment, is a possible antidote when the cost channel is active. The bottom line is that either BM's or EH's proposals to the resolution of the instability under learning cannot be taken for granted when the cost channel matters.

\section{Appendices}

\subsection{Appendix A: Proof of Proposition 1}

The characteristic polynomial of $\Omega$ (given by 6 ) is $P(\xi)=\xi^{2}+A_{1} \xi+A_{0}$ where

$$
\begin{gathered}
A_{0}=\frac{\sigma \beta}{\sigma+\phi_{x}+\left(\kappa_{x}-\kappa \delta \sigma\right) \phi_{\pi}} \\
A_{1}=\frac{-\sigma(1+\beta)-\beta \phi_{x}+\kappa_{x}\left(\phi_{\pi}-1\right)-\left[\kappa \delta \phi_{x}+\left(\kappa_{x}-\kappa \delta \sigma\right) \phi_{\pi}\right]}{\sigma+\phi_{x}+\left(\kappa_{x}-\kappa \delta \sigma\right) \phi_{\pi}}
\end{gathered}
$$

Both eigenvalues of $\Omega$ are inside the unit circle if and only if both of the following conditions hold

$$
\begin{gathered}
\left|A_{0}\right|<1 \\
\left|A_{1}\right|<1+A_{0} .
\end{gathered}
$$

We can note that condition (A3) is always true, whereas condition (A4) implies (7) and (8).

\subsection{Appendix B: Proof of Proposition 2}

Using results of Evans and Honkapohja (2001, chapter 10), E-stability needs that the eigenvalues of $\rho \Omega$ ( $\Omega$ is given by equation. 6$)$ to have real parts less than one. The eigenvalues of $\rho \Omega$ are given by the product of the eigenvalues of $\Omega$ and $\rho$, and since $0<\rho<1$, it suffices that eigenvalues of $B$ to have real parts less than 1. On the other hand, the MSV solution will not be E-stable if any eigenvalue of $\Omega$ has a real part greater than 1 . The characteristic polynomial of $\Omega-I$ (where $I$ is a corresponding identity matrix) given by $P(\xi)=\xi^{2}+A_{1} \xi+A_{0}$ where

$$
A_{1}=\frac{\sigma(1-\beta)+\left(\kappa_{x}-\delta \kappa \sigma\right) \phi_{\pi}+\phi_{x}+\kappa_{x}\left(\phi_{\pi}-1\right)+(1-\beta-\delta \kappa) \phi_{x}}{\sigma+\phi_{x}+\left(\kappa_{x}-\delta \kappa \sigma\right) \phi_{\pi}}
$$




$$
A_{0}=\frac{(1-\beta-\delta \kappa) \phi_{x}+\kappa_{x}\left(\phi_{\pi}-1\right)}{\sigma+\phi_{x}+\left(\kappa_{x}-\delta \kappa \sigma\right) \phi_{\pi}}
$$

It is necessary for both eigenvalues of $\Omega-I$ to have negative real parts. According to the Routh Theorem, that condition holds if and only if $A_{1}>0$ and $A_{0}>0$. We can note that

$$
A_{1}=A_{0}+\frac{\sigma(1-\beta)+\left(\kappa_{x}-\delta \kappa \sigma\right) \phi_{\pi}+\phi_{x}}{\sigma+\phi_{x}+\left(\kappa_{x}-\delta \kappa \sigma\right) \phi_{\pi}}
$$

Thus, given that $\kappa_{x}-\delta \kappa \sigma$ equals $\kappa \eta$ when $\delta=1, A_{0}>0$ implies $A_{1}>0$. Hence, the E-stability condition, given by (9), is derived from $A_{0}>0$.

\subsection{Appendix C: Proof of Proposition 3}

The characteristic polynomial of $\Omega$ (given by 11) is $P(\xi)=\xi^{2}+A_{1} \xi+A_{0}$ where

$$
\begin{gathered}
A_{0}=\frac{\beta\left(\sigma-\phi_{x}\right)+\delta \kappa\left(\sigma \phi_{\pi}-\phi_{x}\right)}{\sigma} \\
A_{1}=\frac{\phi_{x}+\kappa_{x}\left(\phi_{\pi}-1\right)-\sigma(\beta+1)-\delta \kappa \sigma \phi_{\pi}}{\sigma}
\end{gathered}
$$

Both eigenvalues of $\Omega$ are inside the unit circle if and only if conditions (A3) and (A4) hold. We can note that condition (A3) implies (12) and (13), whereas condition (A4) implies (14) and (15).

\subsection{Appendix D: Proof of Proposition 4}

As in the previous case, E-stability conditions are given by analyzing the characteristic polynomial of $\Omega-I$ (where $\Omega$ is given by 11 ) given by $P(\xi)=\xi^{2}+A_{1} \xi+A_{0}$ where

$$
\begin{gathered}
A_{1}=\frac{\left(\kappa_{x}-\delta \kappa \sigma\right) \phi_{\pi}+\phi_{x}+\sigma(1-\beta)-\kappa_{x}}{\sigma} \\
A_{0}=\frac{\phi_{x}(1-\beta-\delta \kappa)+\kappa_{x}\left(\phi_{\pi}-1\right)}{\sigma}
\end{gathered}
$$

are necessary for both eigenvalues of $\Omega-I$ to have negative real parts. According to the Routh Theorem, that condition holds if and only if $A_{1}>0$ and $A_{0}>0$. We can note

$$
A_{1}=A_{0}+\frac{\sigma(1-\beta)+(\beta+\kappa \delta) \phi_{x}-\kappa \delta \sigma \phi_{\pi}}{\sigma}
$$

Different from the case of contemporaneous data, $A_{0}>0$ does not imply $A_{1}>0$. In this case, the first E-stability condition, given by (16), is derived from $A_{0}>0$. The second E-stability 
condition, given by (17), is derived from $A_{1}>0$ (using equation D3), provided that $A_{0}>0$.

\subsection{Appendix E: Proof of Proposition 5}

The characteristic polynomial of $\Omega$ (given by 25) has one eigenvalue equal to zero and the other equal to (after replacing $\kappa_{x}$ and $\kappa_{o}$ and making $\delta=1$ )

$$
r=\frac{\lambda \beta+\lambda \kappa-\kappa^{2} \sigma \eta}{\lambda+\kappa^{2} \eta^{2}}
$$

Determinacy requires that $|r|<1$ and thus the latter condition implies

$$
\frac{\kappa^{2} \eta(\sigma-\eta)}{(\beta+\kappa+1)}<\lambda<\frac{\kappa^{2} \eta(\eta+\sigma)}{(\beta+\kappa-1)}
$$

\subsection{Appendix F: Proof of Proposition 6}

E-stability is guaranteed if and only if all of the eigenvalues of $\Omega-I$ (where $\Omega$ is given by 25) have negative real parts. The characteristic polynomial of $\Omega-I$ is given by $\rho(\xi)=\xi^{2}+A_{1} \xi+A_{0}$ where

$$
\begin{gathered}
A_{1}=1+\frac{\lambda+\kappa^{2} \eta^{2}+\kappa^{2} \eta \sigma-\lambda \beta-\lambda \kappa}{\lambda+\kappa^{2} \eta^{2}} \\
A_{0}=\frac{\lambda+\kappa^{2} \eta^{2}+\kappa^{2} \eta \sigma-\lambda \beta-\lambda \kappa}{\lambda+\kappa^{2} \eta^{2}}
\end{gathered}
$$

It is necessary for both eigenvalues of $\Omega-I$ to have negative real parts. According to the Routh Theorem, that condition holds if and only if $A_{1}>0$ and $A_{0}>0$. We can note

$$
A_{1}=A_{0}+1
$$

Thus, a sufficient condition for E-stability is that $A_{0}>0$ implying

$$
\lambda<\frac{\kappa^{2} \eta(\eta+\sigma)}{(\beta+\kappa-1)}
$$

\subsection{Appendix G: Proof of Proposition 8}

We apply the E-stability conditions derived in Evans and Honkapohja (2001, section 10.2.1):

$$
\begin{gathered}
D T_{a}=\Omega(1+\bar{b}) \\
D T_{b}=\bar{b}^{\prime} \otimes \Omega+I \otimes \Omega \bar{b}
\end{gathered}
$$




$$
D T_{c}=\rho^{\prime} \otimes \Omega+I \otimes \Omega \bar{b}
$$

E-stability follows if all real parts of the eigenvalues of $D T_{a}, D T_{b}$ and $D T_{c}$ are lower than 1 . A first necessary condition is that $D T_{a}-I$ has eigenvalues with negative real parts, which is equivalent to $\operatorname{tr}\left(D T_{a}-I\right)<0$ and $\operatorname{det}\left(D T_{a}-I\right)>0$.

$$
D T_{a}-I=\left[\begin{array}{cc}
-\frac{\sigma^{-1}\left(\sigma^{2} \beta \delta_{o}-\beta \kappa_{o}\left(\kappa_{x}+\sigma \beta\right)\right)+\left(\beta \kappa_{o}-\sigma \delta_{o}\right)}{\left(\beta \kappa_{o}-\sigma \delta_{o}\right)} & \frac{\beta \lambda^{-1} \kappa_{o}^{2} \kappa_{x}\left(\bar{b}_{\varphi}+1\right)-\sigma^{-1} \bar{b}_{\pi}\left(\sigma^{2} \beta \delta_{o}-\beta \kappa_{o}\left(\kappa_{x}+\sigma \beta\right)\right)}{\left(\beta \kappa_{o}-\sigma \delta_{o}\right)} \\
\frac{\sigma^{-1} \beta \lambda}{\left(\beta \kappa_{o}-\sigma \delta_{o}\right)} & \frac{\beta \kappa_{o}\left(\bar{b}_{\varphi}+1\right)+\sigma^{-1} \beta \lambda \bar{b}_{\pi}-\left(\beta \kappa_{o}-\sigma \delta_{o}\right)}{\left(\beta \kappa_{o}-\sigma \delta_{o}\right)}
\end{array}\right]
$$

where $\bar{b}_{\pi}$ and $\bar{b}_{\varphi}$ are the coefficients of the MSV at the REE. The determinant of $D T_{a}-I$ is given by the following expression

$$
\operatorname{det}\left(D T_{a}-I\right)=\frac{1}{\sigma^{2} \delta \kappa-\sigma \beta \kappa \eta}\left(\sigma^{2} \kappa(1-\beta)+\beta \kappa \eta \kappa_{x}+\beta \lambda \bar{b}_{\pi}+\sigma \beta \kappa \eta \bar{b}_{\varphi}(1-\beta)\right)
$$

Which is positive iff $(\delta=1) \sigma>\beta \eta$ since $0<\beta<1, \bar{b}_{\pi}>0$ and $\bar{b}_{\varphi}>0$. Notice that without the cost channel $(\delta=0)$ the expression is always negative and therefore $D T_{a}-I$ is an unstable matrix (see EH 2006). The trace of $D T_{a}-I$ can be written as

$$
\operatorname{tr}\left(D T_{a}-I\right)=\frac{1}{(\beta \eta-\sigma \delta)}\left(\sigma(2-\beta)+\beta \eta \bar{b}_{\varphi}+\sigma^{-1} \kappa^{-1} \beta \lambda \bar{b}_{\pi}+\sigma^{-1} \beta \kappa \eta^{2}+\beta \eta(\beta+\kappa-1)\right)
$$

and is always negative if and only if $(\delta=1) \sigma>\beta \eta$. When the cost channel is absent $(\delta=0)$, the trace is always positive and $D T_{a}-I$ is an unstable matrix (see EH 2006).

We now turn to analyze the rest of matrices,

$$
D T_{b}-I=\left[\begin{array}{cc}
\Delta_{11} & 0 \\
\Delta_{21} & \Delta_{22}
\end{array}\right]
$$

where:

$$
\begin{aligned}
& \Delta_{11}=\left[\begin{array}{cc}
-1 & \frac{\beta\left(\bar{b}_{\pi}\left(\beta+\delta_{o}\right) \lambda+\bar{b}_{\varphi} \delta_{o} \kappa_{o} \sigma\right)}{\beta\left(\kappa_{o}^{2}+\lambda\right)+\delta_{o}^{2} \sigma^{2}} \\
0 & -\frac{\bar{b}_{\pi} \beta^{2} \lambda+\delta_{o}^{2} \sigma^{2}+\beta\left(\kappa_{o}^{2}+\lambda+\bar{b}_{\pi} \delta_{o} \lambda+\bar{b}_{\varphi} \delta_{o} \kappa_{o} \sigma\right)}{\beta\left(\kappa_{o}^{2}+\lambda\right)+\delta_{o}^{2} \sigma^{2}}
\end{array}\right] \\
& \Delta_{21}=\left[\begin{array}{cc}
\frac{\beta \bar{b}_{\pi}\left(\beta+\delta_{o}\right) \lambda}{\beta\left(\kappa_{o}^{2}+\lambda\right)+\delta_{o}^{2} \sigma^{2}} & \frac{\beta \bar{b}_{\pi} \delta_{o} \kappa_{o} \sigma}{\beta\left(\kappa_{o}^{2}+\lambda\right)+\delta_{o}^{2} \sigma^{2}} \\
\frac{-\beta \bar{b}_{\pi}\left(\beta+\delta_{o}\right) \lambda}{\beta\left(\kappa_{o}^{2}+\lambda\right)+\delta_{o}^{2} \sigma^{2}} & \frac{-\beta \bar{b}_{\pi} \delta_{o} \kappa_{o} \sigma}{\beta\left(\kappa_{o}^{2}+\lambda\right)+\delta_{o}^{2} \sigma^{2}}
\end{array}\right] \\
& \Delta_{22}=\left[\begin{array}{cc}
-1+\frac{\beta\left(\beta+\delta_{o}\right) \lambda \rho}{\beta\left(\kappa_{o}^{2}+\lambda\right)+\delta_{o}^{2} \sigma^{2}} & \frac{\beta\left(\bar{b}_{\pi}\left(\beta+\delta_{o}\right) \lambda+\left(\bar{b}_{\varphi}+\rho\right) \delta_{o} \kappa_{o} \sigma\right)}{\beta\left(\kappa_{o}^{2}+\lambda\right)+\delta_{o}^{2} \sigma^{2}} \\
\frac{-\beta\left(\beta+\delta_{o}\right) \lambda \rho}{\beta\left(\kappa_{o}^{2}+\lambda\right)+\delta_{o}^{2} \sigma^{2}} & -\frac{\bar{b}_{\pi} \beta^{2} \lambda+\delta_{o}^{2} \sigma^{2}+\beta\left(\kappa_{o}^{2}+\lambda+\bar{b}_{\pi} \delta_{o} \lambda+\left(\bar{b}_{\varphi}+\rho\right) \delta_{o} \kappa_{o} \sigma\right)}{\beta\left(\kappa_{o}^{2}+\lambda\right)+\delta_{o}^{2} \sigma^{2}}
\end{array}\right]
\end{aligned}
$$


The characteristic polynomial of $D T_{b}-I$ is given by $P(\xi)=\xi^{4}+A_{3} \xi^{3}+A_{2} \xi^{2}+A_{1} \xi+A_{0}$. It can be shown that two of roots (e.g. $\xi_{1}$ and $\xi_{2}$ ) are equal to -1 . The rest of roots are given by the following expressions,

$$
\begin{aligned}
\xi_{3} & =\frac{-\beta \kappa_{o}^{2}-\beta^{2} \lambda\left(1-\bar{b}_{\varphi}\right)-\beta \lambda\left(1-\bar{b}_{\varphi} \beta\right)-\bar{b}_{\pi} \beta \delta_{o} \lambda-\delta_{o} \lambda \beta\left(1-\bar{b}_{\varphi}\right)-2 \bar{b}_{\varphi} \beta \delta_{o} \kappa_{o} \sigma-\delta_{o}^{2} \sigma^{2}}{\beta \kappa_{o}^{2}+\beta \lambda+\delta_{o}^{2} \sigma^{2}} \\
\xi_{4} & =\frac{-\beta \kappa_{o}^{2}-\beta \lambda-\bar{b}_{\pi} \beta^{2} \lambda-\bar{b}_{\pi} \beta \delta_{o} \lambda-\bar{b}_{\varphi} \beta \delta_{o} \kappa_{o} \sigma-\delta_{o}^{2} \sigma^{2}}{\beta \kappa_{o}^{2}+\beta \lambda+\delta_{o}^{2} \sigma^{2}}
\end{aligned}
$$

and are always negative since $0<\bar{b}_{\varphi}<1,0<\bar{b}_{\pi}$ and $0<\beta<1$. It follows the $D T_{b}-I$ is a stable matrix.

Finally, we analyze the stability of $D T_{c}-I$.

$$
D T_{c}-I=\left[\begin{array}{cc}
\Delta_{11} & 0 \\
0 & \Delta_{22}
\end{array}\right]
$$

where:

$$
\begin{aligned}
& \Delta_{11}=\left[\begin{array}{cc}
-1 & \frac{\beta\left(\bar{b}_{\pi}\left(\beta+\delta_{o}\right) \lambda+\bar{b}_{\varphi} \delta_{o} \kappa_{o} \sigma\right)}{\beta\left(\kappa_{o}^{2}+\lambda\right)+\delta_{o}^{2} \sigma^{2}} \\
0 & -\frac{\bar{b}_{\pi} \beta^{2} \lambda+\delta_{o}^{2} \sigma^{2}+\beta\left(\kappa_{o}^{2}+\lambda+\bar{b}_{\pi} \delta_{o} \lambda+\bar{b}_{\varphi} \delta_{o} \kappa_{o} \sigma\right)}{\beta\left(\kappa_{o}^{2}+\lambda\right)+\delta_{o}^{2} \sigma^{2}}
\end{array}\right] \\
& \Delta_{22}=\left[\begin{array}{cc}
-1+\frac{\beta\left(\beta+\delta_{o}\right) \lambda \rho}{\beta\left(\kappa_{o}^{2}+\lambda\right)+\delta_{o}^{2} \sigma^{2}} & \frac{\beta\left(\bar{b}_{\pi}\left(\beta+\delta_{o}\right) \lambda+\left(\bar{b}_{\varphi}+\rho\right) \delta_{o} \kappa_{o} \sigma\right)}{\beta\left(\kappa_{o}^{2}+\lambda\right)+\delta_{o}^{2} \sigma^{2}} \\
\frac{-\beta\left(\beta+\delta_{o}\right) \lambda \rho}{\beta\left(\kappa_{o}^{2}+\lambda\right)+\delta_{o}^{2} \sigma^{2}} & -\frac{\bar{b}_{\pi} \beta^{2} \lambda+\delta_{o}^{2} \sigma^{2}+\beta\left(\kappa_{o}^{2}+\lambda+\bar{b}_{\pi} \delta_{o} \lambda+\left(\bar{b}_{\varphi}+\rho\right) \delta_{o} \kappa_{o} \sigma\right)}{\beta\left(\kappa_{o}^{2}+\lambda\right)+\delta_{o}^{2} \sigma^{2}}
\end{array}\right]
\end{aligned}
$$

The characteristic polynomial $D T_{c}-I$ has the form of $P(\xi)=\xi^{4}+A_{3} \xi^{3}+A_{2} \xi^{2}+A_{1} \xi+A_{0}$. It can be shown that two of roots (e.g. $\xi_{1}$ and $\xi_{2}$ ) are equal to -1 . The rest of roots are given by the following expressions,

$$
\begin{aligned}
\xi_{3} & =\frac{-\beta \kappa_{o}^{2}-\beta \lambda(1-\rho \beta)-\beta^{2} \lambda\left(1-\bar{b}_{\varphi}\right)-\bar{b}_{\pi} \beta \delta_{o} \lambda-\delta_{o} \beta \lambda(1-\rho)-\bar{b}_{\varphi} \beta \delta_{o} \kappa_{o} \sigma-\rho \beta \delta_{o} \kappa_{o} \sigma-\delta_{o}^{2} \sigma^{2}}{\beta \kappa_{o}^{2}+\beta \lambda+\delta_{o}^{2} \sigma^{2}} \\
\xi_{4} & =\frac{-\beta \kappa_{o}^{2}-\beta \lambda-\bar{b}_{\pi} \beta^{2} \lambda-\bar{b}_{\pi} \beta \delta_{o} \lambda-\bar{b}_{\varphi} \beta \delta_{o} \kappa_{o} \sigma-\delta_{o}^{2} \sigma^{2}}{\beta \kappa_{o}^{2}+\beta \lambda+\delta_{o}^{2} \sigma^{2}}
\end{aligned}
$$

and are always negative since $0<\bar{b}_{\varphi}<1,0<\bar{b}_{\pi}, 0<\beta<1$ and $0<\rho<1$. It follows the $D T_{c}-I$ is a stable matrix. 


\subsection{Appendix H: Proof of Proposition 9}

We use the approach of Woodford (2003, chapter 4) to perform the determinacy analysis. First, the reduced form under (37) is rewritten as,

$$
\left[\begin{array}{c}
E_{t} \varphi_{2, t+1} \\
E_{t} \pi_{t+1} \\
\varphi_{2 L, t+1}
\end{array}\right]=\Upsilon\left[\begin{array}{c}
\varphi_{2, t} \\
\pi_{t} \\
\varphi_{2 L, t}
\end{array}\right]+\text { other }
$$

The system is determinate iff exactly one root lie inside the unit circle and the rest outside the unit circle. As shown in Woodford (2003) the following cases guarantee determinacy:

either (Case I)

$$
1+A_{2}+A_{1}+A_{0}<0, \quad-1+A_{2}-A_{1}+A_{0}>0
$$

or (Case II):

$$
\begin{gathered}
1+A_{2}+A_{1}+A_{0}>0 ; \quad-1+A_{2}-A_{1}+A_{0}<0 \\
A_{0}^{2}-A_{0} A_{2}+A_{1}-1>0
\end{gathered}
$$

(Case III):

$$
\begin{array}{cc}
1+A_{2}+A_{1}+A_{0}>0 ; & -1+A_{2}-A_{1}+A_{0}<0 \\
A_{0}^{2}-A_{0} A_{2}+A_{1}-1<0 ; & \left|A_{2}\right|>3
\end{array}
$$

Let the characteristic equation of the matrix $\Upsilon$ be written in the form

$$
P(\xi)=\xi^{3}+A_{2} \xi^{2}+A_{1} \xi+A_{0}
$$

where

$$
\begin{gathered}
A_{2}=-\frac{\beta\left(\beta \lambda+\delta_{o} \lambda+\delta_{o} \kappa_{o} \sigma\right)\left(\beta \kappa_{o} \kappa_{x}+\beta \lambda+\beta \delta_{o} \kappa_{o} \sigma+\delta_{o}^{2} \sigma^{2}\right)}{\psi} \\
A_{1}=\frac{\left(\beta \kappa_{o} \kappa_{x}+\beta \lambda+\beta \delta_{o} \kappa_{o} \sigma+\delta_{o}^{2} \sigma^{2}\right)\left(\beta \kappa_{o} \kappa_{x}+\beta \lambda+\beta \delta_{o} \kappa_{o} \sigma+\delta_{o}^{2} \sigma^{2}+\beta^{2} \lambda+2 \beta \delta_{o} \lambda+\delta_{o}^{2} \lambda\right)}{\psi} \\
A_{0}=-\frac{\left(\beta \lambda+\delta_{o} \lambda-\delta_{o} \kappa_{x} \sigma+\delta_{o}^{2} \sigma^{2}\right)\left(\beta \kappa_{o} \kappa_{x}+\beta \lambda+\beta \delta_{o} \kappa_{o} \sigma+\delta_{o}^{2} \sigma^{2}\right)}{\psi}
\end{gathered}
$$

where $\psi=2 \beta^{2} \delta_{o}\left(\beta+\delta_{o}\right) \kappa_{o} \lambda \sigma$

In the polynomial $P(\xi), A_{1}$ and $A_{2}$ are always positive and negative, respectively. Note $A_{0}$ is negative iff $\beta \lambda+\delta_{o} \lambda-\delta_{o} \kappa_{x} \sigma+\delta_{o}^{2} \sigma^{2}>0$. After replacing $\delta_{o}$ and $\kappa_{x}$, it turns out that $\lambda>\kappa^{2} \sigma \eta /(\beta+\kappa)$ guarantees $A_{0}$ 's negativity. Note that such condition for $\lambda$ characterizes the optimal REE (see proposition 7 in the main text). Under such assumption, $-1+A_{2}-A_{1}+A_{0}<$ 
0 and then Case I is ruled out.

\subsection{Appendix I: Proof of Proposition 10}

We apply the E-stability conditions provided in Appendix $\mathrm{H}$ to model under (37). A first necessary condition is that $D T_{a}-I$ has eigenvalues with negative real parts, which is equivalent to $\operatorname{tr}\left(D T_{a}-I\right)<0$ and $\operatorname{det}\left(D T_{a}-I\right)>0$.

$$
D T_{a}-I=\left[\begin{array}{cc}
-1+\frac{\beta\left(\beta+\delta_{o}\right) \lambda}{\delta_{o}^{2} \sigma^{2}+\beta\left(\lambda+\kappa_{o}\left(\kappa_{x}+\delta_{o} \sigma\right)\right)} & \frac{\beta\left(\bar{b}_{\pi}\left(\beta+\delta_{o}\right) \lambda-\left(1+\bar{b}_{\varphi}\right) \delta_{o} \kappa_{o} \sigma\right)}{\delta_{o}^{2} \sigma^{2}+\beta\left(\lambda+\kappa_{o}\left(\kappa_{x}+\delta_{o} \sigma\right)\right)} \\
\frac{-\beta\left(\beta+\delta_{o}\right) \lambda}{\delta_{o}^{2} \sigma^{2}+\beta\left(\lambda+\kappa_{o}\left(\kappa_{x}+\delta_{o} \sigma\right)\right)} & -\frac{\bar{b}_{\pi} \beta^{2} \lambda+\delta_{o}^{2} \sigma^{2}+\beta\left(\lambda+\bar{b}_{\pi} \delta_{o} \lambda+\kappa_{o}\left(\kappa_{x}-\bar{b}_{\varphi} \delta_{o} \sigma\right)\right)}{\delta_{o}^{2} \sigma^{2}+\beta\left(\lambda+\kappa_{o}\left(\kappa_{x}+\delta_{o} \sigma\right)\right)}
\end{array}\right]
$$

where $\bar{b}_{\pi}$ and $\bar{b}_{\varphi}$ are the coefficients of the MSV at the REE. The determinant of $D T_{a}-I$ is given by the following expression

$$
\begin{aligned}
\operatorname{det}\left(D T_{a}-I\right)= & \frac{\beta\left[\kappa_{o} \kappa_{x}-\left(\beta+\delta_{o}-1\right) \lambda\right]+\delta_{o}\left[\lambda\left(\beta+\delta_{o}\right)-\kappa_{o} \sigma\right]}{\delta_{o}^{2} \sigma^{2}+\beta\left(\lambda+\kappa_{o}\left(\kappa_{x}+\delta_{o} \sigma\right)\right)}+ \\
& \frac{\lambda \beta\left(\beta+\delta_{o}\right)\left(1-\bar{b}_{\varphi}\right)+\delta_{o} \kappa_{o} \sigma\left(1-\beta \bar{b}_{\varphi}\right)+\delta_{o}^{2} \sigma^{2}}{\delta_{o}^{2} \sigma^{2}+\beta\left(\lambda+\kappa_{o}\left(\kappa_{x}+\delta_{o} \sigma\right)\right)}
\end{aligned}
$$

There two necessary and sufficient conditions for $\operatorname{det}\left(D T_{a}-I\right)$ to be positive.

$$
\begin{aligned}
\kappa_{o} \kappa_{x}-\left(\beta+\delta_{o}-1\right) \lambda & >0 \rightarrow \lambda<\frac{\kappa^{2} \eta(\eta+\sigma)}{(\beta+\kappa-1)} \\
\lambda\left(\beta+\delta_{o}\right)-\kappa_{o} \sigma & >0 \rightarrow \lambda>\frac{\kappa \eta \sigma}{\beta+\kappa}
\end{aligned}
$$

Notice that under the standard new Keynesian framework $\delta_{o}$ is zero and $\operatorname{det}\left(D T_{a}-I\right)$ is always positive for all parameters value (see EH 2006).

The trace of $D T_{a}-I$ can be written as

$\operatorname{tr}\left(D T_{a}-I\right)=\frac{-\beta\left[\kappa_{o} \kappa_{x}-\left(\beta+\delta_{o}-1\right) \lambda\right]-\bar{b}_{\pi} \beta\left(\beta+\delta_{o}\right) \lambda-2 \delta_{o}^{2} \sigma^{2}-\beta\left(\lambda+\kappa_{o} \kappa_{x}\right)-\beta \kappa_{o} \delta_{o} \sigma\left(1-\bar{b}_{\varphi}\right)}{\delta_{o}^{2} \sigma^{2}+\beta\left(\lambda+\kappa_{o}\left(\kappa_{x}+\delta_{o} \sigma\right)\right)}$

which is always negative iff

$$
\kappa_{o} \kappa_{x}-\left(\beta+\delta_{o}-1\right) \lambda>0 \rightarrow \lambda<\frac{\kappa^{2} \eta(\eta+\sigma)}{(\beta+\kappa-1)}
$$

Again, under the standard new Keynesian framework $\delta_{o}$ is zero and $\operatorname{tr}\left(D T_{a}-I\right)$ is always negative regardless the parametrization (see EH 2006). 
We now turn to the rest of matrices,

$$
D T_{b}-I=\left[\begin{array}{cc}
\Delta_{11} & 0 \\
\Delta_{21} & \Delta_{22}
\end{array}\right]
$$

where:

$$
\begin{aligned}
& \Delta_{11}=\left[\begin{array}{cc}
-1 & \frac{\beta\left(\bar{b}_{\pi}\left(\beta+\delta_{o}\right) \lambda-\bar{b}_{\varphi} \delta_{o} \kappa_{o} \sigma\right)}{\delta_{o}^{2} \sigma^{2}+\beta\left(\lambda+\kappa_{o}\left(\kappa_{x}+\delta_{o} \sigma\right)\right)} \\
0 & -\frac{\bar{b}_{\pi} \beta^{2} \lambda+\delta_{o}^{2} \sigma^{2}+\beta\left(\lambda+\bar{b}_{\pi} \delta_{o} \lambda+\kappa_{o}\left(\kappa_{x}+\delta_{o} \sigma-\bar{b}_{\varphi} \delta_{o} \sigma\right)\right)}{\delta_{o}^{2} \sigma^{2}+\beta\left(\lambda+\kappa_{o}\left(\kappa_{x}+\delta_{o} \sigma\right)\right)}
\end{array}\right] \\
& \Delta_{21}=\left[\begin{array}{cc}
\frac{\beta \bar{b}_{\pi}\left(\beta+\delta_{o}\right) \lambda}{\delta_{o}^{2} \sigma^{2}+\beta\left(\lambda+\kappa_{o}\left(\kappa_{x}+\delta_{o} \sigma\right)\right)} & \frac{-\beta \bar{b}_{\pi} \delta_{o} \kappa_{o} \sigma}{\delta_{o}^{2} \sigma^{2}+\beta\left(\lambda+\kappa_{o}\left(\kappa_{x}+\delta_{o} \sigma\right)\right)} \\
\frac{-\beta \bar{b}_{\pi}\left(\beta+\delta_{o}\right) \lambda}{\beta \delta_{o} \sigma^{2}+\beta\left(\lambda+\kappa_{o}\left(\kappa_{x}+\delta_{o} \sigma\right)\right)} & \frac{\beta \bar{b}_{o} \delta_{o} \sigma}{\delta_{o}^{2} \sigma^{2}+\beta\left(\lambda+\kappa_{o}\left(\kappa_{x}+\delta_{o} \sigma\right)\right)}
\end{array}\right] \\
& \Delta_{22}=\left[\begin{array}{cc}
-1+\frac{\beta\left(\beta+\delta_{o}\right) \lambda \bar{b}_{\varphi}}{\delta_{o}^{2} \sigma^{2}+\beta\left(\lambda+\kappa_{o}\left(\kappa_{x}+\delta_{o} \sigma\right)\right)} & \frac{\beta\left(\bar{b}_{\pi}\left(\beta+\delta_{o}\right) \lambda-2 \bar{b}_{\varphi} \delta_{o} \kappa_{o} \sigma\right)}{\delta_{o}^{2} \sigma^{2}+\beta\left(\lambda+\kappa_{o}\left(\kappa_{x}+\delta_{o} \sigma\right)\right)} \\
\frac{-\beta\left(\beta+\delta_{o}\right) \lambda \bar{b}_{\varphi}}{\delta_{o}^{2} \sigma^{2}+\beta\left(\lambda+\kappa_{o}\left(\kappa_{x}+\delta_{o} \sigma\right)\right)} & -\frac{\bar{b}_{\pi} \beta^{2} \lambda+\delta_{o}^{2} \sigma^{2}+\beta\left(\lambda+\bar{b}_{\pi} \delta_{o} \lambda+\kappa_{o}\left(\kappa_{x}+\delta_{o} \sigma-2 \bar{b}_{\varphi} \delta_{o} \sigma\right)\right)}{\delta_{o}^{2} \sigma^{2}+\beta\left(\lambda+\kappa_{o}\left(\kappa_{x}+\delta_{o} \sigma\right)\right)}
\end{array}\right]
\end{aligned}
$$

The characteristic polynomial of $D T_{b}-I$ is given by $P(\xi)=\xi^{4}+A_{3} \xi^{3}+A_{2} \xi^{2}+A_{1} \xi+A_{0}$. It can be shown that two of roots (e.g. $\xi_{1}$ and $\xi_{2}$ ) are equal to -1 . The rest of roots are given by the following expressions,

$$
\begin{aligned}
\xi_{3} & =\frac{-\beta \kappa_{o} \kappa_{x}-\beta \lambda-\bar{b}_{\pi} \beta^{2} \lambda-\bar{b}_{\pi} \beta \delta_{o} \lambda-\beta \delta_{o} \kappa_{o} \sigma\left(1-\bar{b}_{\varphi}\right)-\delta_{o}^{2} \sigma^{2}}{\delta_{o}^{2} \sigma^{2}+\beta\left(\lambda+\kappa_{o}\left(\kappa_{x}+\delta_{o} \sigma\right)\right)} \\
\xi_{4} & =\frac{-\beta\left[\kappa^{2} \eta^{2}+(1-\beta) \lambda\right]-\delta_{o}^{2} \lambda-2 \beta\left(\lambda\left(\beta+\delta_{o}\right)+\delta_{o} \kappa_{o} \sigma\right)\left(1-\bar{b}_{\varphi}\right)-\delta_{o}^{2} \sigma^{2}}{\delta_{o}^{2} \sigma^{2}+\beta\left(\lambda+\kappa_{o}\left(\kappa_{x}+\delta_{o} \sigma\right)\right)}
\end{aligned}
$$

and are always negative since $0<\bar{b}_{\varphi}<1,0<\bar{b}_{\pi}$ and $0<\beta<1$. It follows the $D T_{b}-I$ is a stable matrix.

Finally, we analyze the stability of $D T_{c}-I$.

$$
D T_{c}-I=\left[\begin{array}{cc}
\Delta_{11} & 0 \\
0 & \Delta_{22}
\end{array}\right]
$$

where:

$$
\begin{aligned}
& \Delta_{11}=\left[\begin{array}{cc}
-1 & \frac{\beta\left(\bar{b}_{\pi}\left(\beta+\delta_{o}\right) \lambda-\bar{b}_{\varphi} \delta_{o} \kappa_{o} \sigma\right)}{\delta_{o}^{2} \sigma^{2}+\beta\left(\lambda+\kappa_{o}\left(\kappa_{x}+\delta_{o} \sigma\right)\right)} \\
0 & -\frac{\bar{b}_{\pi} \beta^{2} \lambda+\delta_{o}^{2} \sigma^{2}+\beta\left(\lambda+\bar{b}_{\pi} \delta_{o} \lambda+\kappa_{o}\left(\kappa_{x}+\delta_{o} \sigma-\bar{b}_{\varphi} \delta_{o} \sigma\right)\right)}{\delta_{o}^{2} \sigma^{2}+\beta\left(\lambda+\kappa_{o}\left(\kappa_{x}+\delta_{o} \sigma\right)\right)}
\end{array}\right] \\
& \Delta_{22}=\left[\begin{array}{cc}
-1+\frac{\beta\left(\beta+\delta_{o}\right) \lambda \rho}{\delta_{o}^{2} \sigma^{2}+\beta\left(\lambda+\kappa_{o}\left(\kappa_{x}+\delta_{o} \sigma\right)\right)} & \frac{\beta\left(\bar{b}_{\pi}\left(\beta+\delta_{o}\right) \lambda-\left(\bar{b}_{\varphi}+\rho\right) \delta_{o} \kappa_{o} \sigma\right)}{\delta_{o}^{2} \sigma^{2}+\beta\left(\lambda+\kappa_{o}\left(\kappa_{x}+\delta_{o} \sigma\right)\right)} \\
\frac{-\beta\left(\beta+\delta_{o}\right) \lambda \rho}{\delta_{o}^{2} \sigma^{2}+\beta\left(\lambda+\kappa_{o}\left(\kappa_{x}+\delta_{o} \sigma\right)\right)} & -\frac{\bar{b}_{\pi} \beta^{2} \lambda+\delta_{o} \sigma^{2}+\beta\left(\lambda+\bar{b}_{\pi} \delta_{o} \lambda+\kappa_{o}\left(\kappa_{x}-\delta_{o} \sigma\left(-1+\bar{b}_{\varphi}+\rho\right)\right)\right)}{\delta_{o}^{2} \sigma^{2}+\beta\left(\lambda+\kappa_{o}\left(\kappa_{x}+\delta_{o} \sigma\right)\right)}
\end{array}\right]
\end{aligned}
$$


The characteristic polynomial $D T_{c}-I$ has the form of $P(\xi)=\xi^{4}+A_{3} \xi^{3}+A_{2} \xi^{2}+A_{1} \xi+A_{0}$. It can be shown that two of roots (e.g. $\xi_{1}$ and $\xi_{2}$ ) are equal to -1 . The rest of roots are given by the following expressions,

$$
\begin{aligned}
\xi_{3} & =\frac{-\beta \kappa_{o} \kappa_{x}-\beta \lambda-\bar{b}_{\pi} \beta^{2} \lambda-\bar{b}_{\pi} \beta \delta_{o} \lambda-\beta \delta_{o} \kappa_{o} \sigma\left(1-\bar{b}_{\varphi}\right)-\delta_{o}^{2} \sigma^{2}}{\delta_{o}^{2} \sigma^{2}+\beta\left(\lambda+\kappa_{o}\left(\kappa_{x}+\delta_{o} \sigma\right)\right)} \\
\xi_{4} & =\frac{-\beta\left[\kappa^{2} \eta^{2}+(1-\rho \beta) \lambda+\delta_{o} \lambda(1-\rho)\right]-\delta_{o}^{2} \lambda-\beta \lambda\left(\beta+\delta_{o}\right)\left(1-\bar{b}_{\varphi}\right)-\beta \delta_{o} \kappa_{o} \sigma\left(2-\rho-\bar{b}_{\varphi}\right)-\delta_{o}^{2} \sigma^{2}}{\delta_{o}^{2} \sigma^{2}+\beta\left(\lambda+\kappa_{o}\left(\kappa_{x}+\delta_{o} \sigma\right)\right)}
\end{aligned}
$$

and are always negative since $0<\bar{b}_{\varphi}<1,0<\bar{b}_{\pi}, 0<\beta<1$ and $0<\rho<1$. It follows the $D T_{c}-I$ is a stable matrix.

\section{References}

[1] Barth, M. and V. Ramey (2001), "The Cost Channel of Monetary Transmission", NBER Macroeconomics Annual, 199-239.

[2] Benhabib, J., S. Schmitt-Grohe and M. Uribe (2001), "Monetary policy and multiple equilibria", The American Economic Review, 91, pp. 167-186.

[3] Blanchard, Olivier J. and Charles M. Kahn (1980), "The Solution of Linear Difference Models under Rational Expectations", Econometrica, 48/5, pp 1305-1312.

[4] Brückner, M. and A. Schabert (2003), "Supply-side Effects of Monetary Policy and Equilibrium Multiplicity", Economic Letters, 79, pp. 205-211.

[5] Bullard, J. (2006) "The Learnability Criterion and Monetary Policy", mimeo, St. Louis Federal Reserve.

[6] Bullard, J. and K. Mitra (2002), "Learning about Monetary Policy Rules", Journal of Monetary Economics, 49, pp. 1105-1129.

[7] Bullard, J. and K. Mitra (2006), "Determinacy, Learnability and Monetary Policy Inertia", Forthcoming in Journal of Money, Credit and Banking.

[8] Calvo, G. (1983), "Staggered Prices in a Utility Maximizing Framework". Journal of Monetary Economics, 12, pp. 383-398.

[9] Chowdhury, I., M. Hoffmann and A. Schabert (2006), "Inflation Dynamics and the Cost Channel of Monetary Transmission", European Economic Review 50, pp. 995-1016. 
[10] Christiano, L., M. Eichenbaum and C. Evans (2005), "Nominal Rigidities and the Dynamics Effects of a Shock to a Monetary Policy", Journal of Political Economy, 113 (1), pp $1-45$

[11] Clarida, R., Gali, J. and M. Gertler (2000), "Monetary Policy Rules and Macroeconomic Stability: Evidence and Some Theory", Quarterly Journal of Economics, 115, pp 147-180.

[12] Duffy, J. and W. Xiao (2005), "The Value of Interest Rate Stabilization Policies When Agents are Learning", forthcoming Journal of Money Credit and Banking.

[13] Evans, G. and S. Honkapohja (1999), "Learning Dynamics", in (Taylor and Woodford 1999), Chapter 7, pp.449-542.

[14] Evans, G. and S. Honkapohja (2001), Learning and Expectations in Macroeconomics, Princeton University Press, Princeton.

[15] Evans, G. and S. Honkapohja (2003), "Expectations and Stability Problem for Optimal Monetary Policies" Review of Economic Studies 70, 807-824

[16] Evans, G. and S. Honkapohja (2006), "Monetary Policy, Expectations and Commitment", Scandinavian Journal of Economics, 108(1), pp.15-38.

[17] Honkapohja, S., K. Mitra and G. Evans (2003), "Notes on Agent's Behavioral Rules Under Adaptive Learning and Recent Studies of Monetary Policy", mimeo, University of Helsinki.

[18] Kurozomi, T. (2006), "Determinacy and expectational stability of equilibrium in a monetary sticky-price model with Taylor rule", Journal of Monetary Economics 53, pp. 827-846

[19] McCallum, B. T. (1983), "On non-uniqueness in rational expectations models: An attempt at perspective", Journal of Monetary Economics 11, pp 139-168.

[20] McCallum, B. T. and E. Nelson (1999), "Performance of Operational Policy Rules in and Estimated Semi-classical Model", in J. Taylor (ed.) Monetary Policy Rules, University of Chicago Press, Chicago, pp. 15-45.

[21] McCallum, B. T., (2007), "E-stability vis-à-vis determinacy results for a broad class of linear rational expectations models, Journal of Economic Dynamics and Control, 31, pp. 1376-1391.

[22] Preston, B. (2005), "Learning about Monetary Policy Rules when Long-Horizon Forecasts Matters", International Journal of Central Banking, Vol. 1(2), pp 81-126. 
[23] Ravenna F. and C. Walsh (2006), "Optimal Monetary Policy with the Cost Channel", Journal of Monetary Economics, 53 pp. 889-911.

[24] Surico, P. (2006), "The Cost Channel of Monetary Policy and Indeterminacy", mimeo, University of Bari.

[25] Taylor, J. (1993), "Discretion versus Policy Rules in Practice", Carnegie-Rochester Series on Public Policy, 39, pp 195-214.

[26] Taylor, J. and M. Woodford (Eds.) (1999), Handbook of Macroeconomics, Volume 1, Elsevier, Amsterdam.

[27] Woodford, M. (1999), "Optimal Monetary Policy Inertia", The Manchester School, Supplement 67, pp 1-35.

[28] Woodford, M. (2003), Interest and Prices: Foundations of a Theory of Monetary Policy. Princeton University Press, Princeton. 


\section{Documentos de Trabajo publicados \\ Working Papers published}

La serie de Documentos de Trabajo puede obtenerse de manera gratuita en formato pdf en la siguiente dirección electrónica:

http://www.bcrp.gob.pe/

The Working Paper series can be downloaded free of charge in pdf format from:

http://www.bcrp.gob.pe/

2007

\section{Agosto | August}

DT N²007-013

Determinantes del crecimiento económico: Una revisión de la literatura existente y estimaciones para el período 1960-2000

Raymundo Chirinos

DT N²007-012

Independencia Legal y Efectiva del Banco Central de Reserva del Perú

Vicente Tuesta Reátegui

DT N ${ }^{\circ} 2007-011$

Regla Fiscal Estructural y el Ciclo del Producto

Carlos Montoro y Eduardo Moreno

DT N ${ }^{\circ} 2007-010$

Oil Shocks and Optimal Monetary Policy

Carlos Montoro

\section{Mayo I May}

DT N²007-009

Estimación de la Frontera Eficiente para las AFP en el Perú y el Impacto de los Límites de Inversión: 1995 - 2004

Javier Pereda

DT N²007-008

Efficiency of the Monetary Policy and Stability of Central Bank Preferences. Empirical Evidence for Peru

Gabriel Rodríguez

DT $\mathrm{N}^{\circ} 2007-007$

Application of Three Alternative Approaches to Identify Business Cycles in Peru Gabriel Rodríguez

\section{Abril \ April}

DT N²007-006

Monetary Policy in a Dual Currency Environment

Guillermo Felices, Vicente Tuesta 


\section{Marzo I March}

DT $\mathrm{N}^{\circ}$ 2007-005

Monetary Policy, Regime Shift and Inflation Uncertainty in Peru (1949-2006)

Paul Castillo, Alberto Humala, Vicente Tuesta

DT N ${ }^{\circ} 2007-004$

Dollarization Persistence and Individual Heterogeneity

Paul Castillo y Diego Winkelried

DT No 2007-003

Why Central Banks Smooth Interest Rates? A Political Economy Explanation Carlos Montoro

\section{Febrero I February}

DT No2007-002

Comercio y crecimiento: Una revisión de la hipótesis "Aprendizaje por las

Exportaciones"

Raymundo Chirinos Cabrejos

\section{Enero I January}

DT $N^{\circ} 2007-001$

Perú: Grado de inversión, un reto de corto plazo

Gladys Choy Chong

2006

\section{Octubre l October}

DT N²006-010

Dolarización financiera, el enfoque de portafolio y expectativas:

Evidencia para América Latina (1995-2005)

Alan Sánchez

DT No2006-009

Pass-through del tipo de cambio y política monetaria:

Evidencia empírica de los países de la OECD

César Carrera, Mahir Binici

\section{Agosto \ August}

DT No2006-008

Efectos no lineales de choques de política monetaria y de tipo de cambio real en economías parcialmente dolarizadas: un análisis empírico para el Perú

Saki Bigio, Jorge Salas

\section{Junio I June}

DT $\mathrm{N}^{\circ} 2006-007$

Corrupción e Indicadores de Desarrollo: Una Revisión Empírica

Saki Bigio, Nelson Ramírez-Rondán 
DT $\mathrm{N}^{\circ} 2006-006$

Tipo de Cambio Real de Equilibrio en el Perú: modelos BEER y construcción de bandas de confianza

Jesús Ferreyra y Jorge Salas

DT N²006-005

Hechos Estilizados de la Economía Peruana

Paul Castillo, Carlos Montoro y Vicente Tuesta

DT N²006-004

El costo del crédito en el Perú, revisión de la evolución reciente

Gerencia de Estabilidad Financiera

DT N²006-003

Estimación de la tasa natural de interés para la economía peruana

Paul Castillo, Carlos Montoro y Vicente Tuesta

\section{Mayo I May}

DT $\mathrm{N}^{\circ} 2006-02$

El Efecto Traspaso de la tasa de interés y la política monetaria en el Perú: 1995-2004 Alberto Humala

\section{Marzo I March}

DT N²006-01

¿Cambia la Inflación Cuando los Países Adoptan Metas Explícitas de Inflación?

Marco Vega y Diego Winkelreid

2005

\section{Diciembre I December}

DT N²005-008

El efecto traspaso de la tasa de interés y la política monetaria en el Perú 1995-2004 Erick Lahura

\section{Noviembre I November}

DT N²005-007

Un Modelo de Proyección BVAR Para la Inflación Peruana

Gonzalo Llosa, Vicente Tuesta y Marco Vega

DT $\mathrm{N}^{\circ} 2005-006$

Proyecciones desagregadas de la variación del Índice de Precios al Consumidor (IPC), del Índice de Precios al Por Mayor (IPM) y del Crecimiento del Producto Real (PBI)

Carlos R. Barrera Chaupis

\section{Marzo I March}

DT N²005-005

Crisis de Inflación y Productividad Total de los Factores en Latinoamérica Nelson Ramírez Rondán y Juan Carlos Aquino. 
DT N²005-004

Usando información adicional en la estimación de la brecha producto en el Perú: una aproximación multivariada de componentes no observados

Gonzalo Llosa y Shirley Miller.

DT $\mathrm{N}^{\circ} 2005-003$

Efectos del Salario Mínimo en el Mercado Laboral Peruano

Nikita R. Céspedes Reynaga

\section{Enero I January}

DT N²005-002

Can Fluctuations in the Consumption-Wealth Ratio Help to Predict Exchange Rates? Jorge Selaive y Vicente Tuesta

DT N²005-001

How does a Global disinflation drag inflation in small open economies?

Marco Vega y Diego Winkelreid 